\title{
Vibration and transient response of structural-acoustic interior coupled systems with dissipative interface
}

\author{
J.-F. Deü, W. Larbi, R. Ohayon \\ Conservatoire National des Arts et Métiers (CNAM), Structural Mechanics and Coupled Systems Laboratory, Chair of Mechanics, Case 353, 2 Rue Conté, 75003 Paris, France
}

This work concerns the variational formulation and the numerical computation of vibroacoustic interior problems with interface damping. The coupled system consists of an elastic structure (described by a displacement field) containing an inviscid, compressible and barotropic fluid (described by a pressure field), gravity effects being neglected. Within the context of noise reduction techniques, we propose to investigate the effect of introducing a thin layer of damping material at the fluid-structure interface. The originality of this work lies in the introduction of an additional unknown field at the fluid-structure interface, namely the normal fluid displacement field. With this new scalar unknown, various interface damping models can be introduced in the variational formulation. Moreover, the associated finite element matrix system can be written in a symmetric form and directly solved in frequency and time domains.

\author{
Keywords: \\ Elastoacoustic \\ Dissipative interface \\ Noise reduction \\ Finite element method \\ Symmetric formulation \\ Wall impedance
}

\section{Introduction}

In recent years, a considerable amount of research has been devoted to the development and testing of noise reduction techniques by passive damping treatments. Many approaches have been proposed in the literature, in particular to model the absorbing material. These approaches are generally based on poroelastic material modeling [1-4]. In this work, we focuse on the formulation of structural-acoustic problems with interface damping using a local wall impedance approach. In this context, let us mention the paper of Kehr-Candille and Ohayon [5], where a frequencydependent impedance is introduced to describe the absorbing material at the fluid-structure interface, and where a substructuring method is used to solve the dissipative structural-acoustic system. In that work, the fluid is described by a scalar unknown field (pressure or fluid displacement potential) and the problem is numerically solved in frequency domain by the finite element method. Using the same kind of approach, Bermúdez and Rodríguez present in [6] a finite element method to compute the dynamic response of an elastoacoustic system with dissipative interface subject to an external harmonic excitation. In their paper, a displacement formulation is used for both media, requiring a particular attention to the discretization of the admissible class of irrotational motions of the fluid. More recently, an original formulation for internal acoustic dissipative problems, based on the introduction of the normal fluid displacement field at the absorbing walls, has been proposed by the authors [7].

In the present paper, the approach developed by the authors for acoustic problems is extended to structural-acoustic interior problems with interface damping following the preliminary results presented in [8]. The coupled system consists of an elastic structure (described by a displacement field $\mathbf{u}$ ) containing an inviscid, compressible and barotropic fluid (described by a pressure field $p$ ), gravity effects being neglected. In order to take the effect of a thin layer of absorbing material at the fluid-structure interface into account, an additional scalar unknown field, namely the normal fluid displacement field $\eta$, is introduced. With this new scalar unknown, various interface damping models can be introduced in the variational formulation. Moreover, the associated finite element matrix system can be solved in frequency and time domains. Here, a viscoelastic Kelvin-Voigt constitutive equation is used to take into account the dissipation at the fluid-structure interface. For a given material, the damping parameters can be found from acoustic impedance measurements in a particular frequency range [5,9] or from a refined poroelactic approaches based on Biot-Allard theory [1].

The outline of the paper is the following: first, a non-symmetric finite element formulation in terms of $(\mathbf{u}, \eta, p)$ is proposed for harmonic vibrations and transient responses of structural-acoustic problems with interface damping. It is shown that this formulation degenerates into a classical fluid-structure one in terms of $(\mathbf{u}, p)$ for perfect contact. Then, the formulation is written in a symmetric form through the introduction of an intermediate unknown field, 
namely the displacement potential of the fluid $\varphi[10,11]$. Finally, numerical examples are presented in order (i) to validate the new formulation by comparison with modal results given in literature, and (ii) to evaluate the influence of the damping model on the dynamic responses of the structural-acoustic system. In this last case, a direct time integration method and a modal reduction approach are used to compute the transient response of the coupled system.

\section{Finite element formulation for the elastoacoustic problem with interface damping}

Let us consider an elastic structure completely filled with an acoustic fluid and subject to external forces. We propose to investigate the effect of introducing a thin layer of absorbing material (for example porous insulated material) at the fluid-structure interface in order to damp the elastoacoustic energy (see Fig. 1). In the present analysis, we suppose that this physical interface can be modeled by a "smeared-mass" geometric surface. Therefore, this interface will be described by a particular constitutive law through the introduction of a dissipative wall acoustic impedance $Z(\omega)[12]$.

Following the numerical results presented in Refs. [5,6,13] for particular geometries, $Z(\omega)$ can be approximated by a viscoelastic Kelvin-Voigt model (see Fig. 2), i.e. by the sum of a constant real part and an imaginary part depending on the frequency: $Z(\omega)=d^{I}-\mathrm{i}\left(m^{I} \omega-k^{I} / \omega\right)$. The parameters $m^{I}, k^{I}$ and $d^{I}$ are associated respectively to the mass, elastic and viscous contributions of the absorbing layer.

We establish here (i) the variational formulation of the structural-acoustic problem with damping interface using a KelvinVoigt smeared-mass model and (ii) the corresponding matrix equations resulting for instance from a finite element discretization. It is shown that this formulation degenerates into a classical one in the case of perfect interface.

\subsection{Dynamic equations of the structure}

Let us consider an elastic structure occupying the domain $\Omega_{\mathrm{S}}$ at equilibrium. The interior fluid domain is denoted by $\Omega_{\mathrm{F}}$ and the

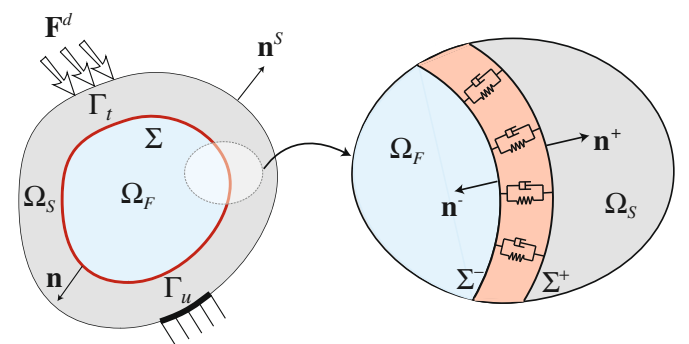

Fig. 1. Fluid-structure problem with interface damping.

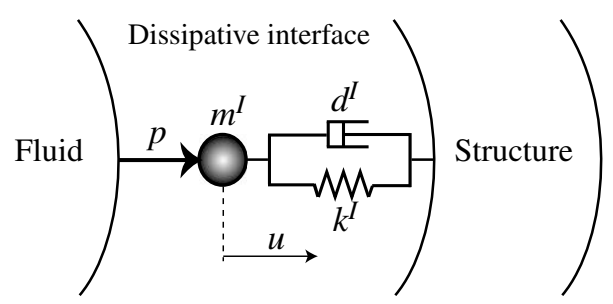

Fig. 2. Fluid-structure dissipative interface. fluid-structure interface, which is firstly considered as a tridimensional domain, is denoted by $\Sigma$ (see Fig. 1 ).

The chosen unknown field in the structure domain is the displacement field $\mathbf{u}^{\mathrm{S}}$ and the associated linearized strain and stress tensors are denoted by $\boldsymbol{\varepsilon}\left(\mathbf{u}^{\mathrm{S}}\right)$ and $\boldsymbol{\sigma}\left(\mathbf{u}^{\mathrm{S}}\right)$, respectively. Moreover, we denote by $\rho_{\mathrm{S}}$ the mass density of the structure and by $\mathbf{n}^{\mathrm{S}}$ the unit normal vector, external to $\Omega_{\mathrm{S}}$.

The local equations describing the response of the structure, fixed on a part of the external boundary $\Gamma_{u}$, and subject to a given force density $\mathbf{F}^{\mathrm{d}}$ on $\Gamma_{t}$, are

$\operatorname{div} \boldsymbol{\sigma}\left(\mathbf{u}^{\mathrm{S}}\right)-\rho_{\mathrm{S}} \frac{\partial^{2} \mathbf{u}^{\mathrm{S}}}{\partial t^{2}}=\mathbf{0}$ in $\Omega_{\mathrm{S}}$,

$\boldsymbol{\sigma}\left(\mathbf{u}^{\mathrm{S}}\right) \mathbf{n}^{\mathrm{S}}=\mathbf{F}^{\mathrm{d}}$ on $\Gamma_{t}$,

$\mathbf{u}^{\mathrm{S}}=\mathbf{0}$ on $\Gamma_{u}$.

Moreover, the continuity conditions between the structure and the interface are given by:

$\boldsymbol{\sigma}\left(\mathbf{u}^{\mathrm{S}}\right) \mathbf{n}^{\mathrm{S}}+\boldsymbol{\sigma}^{I} \mathbf{n}^{+}=\mathbf{0}$ on $\Sigma^{+}$,

$\left(\mathbf{u}^{\mathrm{S}}-\mathbf{u}^{+}\right) \cdot \mathbf{n}^{+}=0$ on $\Sigma^{+}$,

where $\Sigma^{+}$is the interface boundary seen from the structure, $\sigma^{I}$ is the unidimensional stress in the interface domain, $\mathbf{n}^{+}$is the unit normal vector pointing into $\Omega_{\mathrm{S}}$, and $\mathbf{u}^{+}$is the interface displacement field on $\Sigma^{+}$

\subsection{Compressible fluid motion}

Since the compressible fluid is assumed to be inviscid, instead of describing its motion by a fluid displacement vector field $\mathbf{u}^{\mathrm{F}}$, which requires an appropriate discretization of the fluid irrotationality constraint curl $\mathbf{u}^{\mathrm{F}}=\mathbf{0}$, we use the pressure scalar field $p$, which satisfies the classical Helmholtz equation:

$\Delta p-\frac{1}{c^{2}} \frac{\partial^{2} p}{\partial t^{2}}=0$ in $\Omega_{\mathrm{F}}$,

where $c$ is the constant sound speed in the fluid.

The continuity conditions between the fluid and the interface are

$-p \mathbf{n}^{\mathrm{F}}+\sigma^{I} \mathbf{n}^{-}=\mathbf{0}$ on $\Sigma^{-}$,

$\left(\mathbf{u}^{\mathrm{F}}-\mathbf{u}^{-}\right) \cdot \mathbf{n}^{-}=0$ on $\Sigma^{-}$,

where $\Sigma^{-}$is the interface boundary seen from the fluid, $\mathbf{n}^{\mathrm{F}}$ the unit normal vectors external to $\Omega_{\mathrm{F}}, \mathbf{n}^{-}$is the unit normal vector pointing into $\Omega_{\mathrm{F}}$, and $\mathbf{u}^{-}$is the interface displacement field on $\Sigma^{-}$.

Moreover, the linearized Euler equation on the wall $\Sigma^{-}$gives the following relation:

$\nabla p \cdot \mathbf{n}^{\mathrm{F}}=-\rho_{\mathrm{F}} \frac{\partial^{2} \mathbf{u}^{\mathrm{F}}}{\partial t^{2}} \cdot \mathbf{n}^{\mathrm{F}}$ on $\Sigma^{-}$,

where $\rho_{\mathrm{F}}$ is the constant mass density of the fluid at rest.

\subsection{Damping behavior of the fluid-structure interface}

Between the fluid and the structure, we now consider that the interface domain, of mass $m^{I}$, is without thickness $\left(\Sigma=\Sigma^{+}=\Sigma^{-}\right.$ and $\mathbf{n}=\mathbf{n}^{+}=-\mathbf{n}^{-}=\mathbf{n}^{\mathrm{F}}$ ) (see Fig. 1).

For the Kelvin-Voigt model used in this work, the stress within the interface is defined by:

$\sigma^{I}=k^{I} \llbracket \mathbf{u} \rrbracket \cdot \mathbf{n}+d^{I} \llbracket \frac{\partial \mathbf{u}}{\partial t} \rrbracket \cdot \mathbf{n}+m^{I} \llbracket \frac{\partial^{2} \mathbf{u}}{\partial t^{2}} \rrbracket \cdot \mathbf{n}$,

where $k^{I}, d^{I}$ and $m^{I}$ are parameters of the damping model and $\llbracket \cdot \rrbracket=(\cdot)^{+}-(\cdot)^{-}$. As can be seen, the first term is proportional to the normal component of the displacement and accounts the elastic 
behavior of the interface material, the second one is proportional to the normal velocity and models the viscous damping, and the third one is proportional to the normal acceleration and models the smeared-mass effect of the interface.

\subsection{Boundary value problem in terms of $(\mathbf{u}, \eta, p)$}

In order to rewrite all the previous equations in a more compact form, we introduce the fluid normal displacement field at the absorbing interface $\Sigma$ denoted by $\eta=\mathbf{u}^{\mathrm{F}} \cdot \mathbf{n}$. Moreover, to simplify the notations, we replace $\mathbf{u}^{\mathrm{S}}$ by $\mathbf{u}$ in the following. With these notations, the dynamic equations of the coupled fluid-structure problem with interface damping can be written as

- Structure

$$
\begin{aligned}
& \operatorname{div} \boldsymbol{\sigma}(\mathbf{u})=\rho_{\mathrm{S}} \frac{\partial^{2} \mathbf{u}}{\partial t^{2}} \text { in } \Omega_{\mathrm{S}}, \\
& \boldsymbol{\sigma}(\mathbf{u}) \mathbf{n}^{\mathrm{S}}=\mathbf{F}^{\mathrm{d}} \text { on } \Gamma_{t}, \\
& \mathbf{u}=\mathbf{0} \text { on } \Gamma_{u}, \\
& \boldsymbol{\sigma}(\mathbf{u}) \mathbf{n}^{\mathrm{S}}=-\left[k^{I}(\mathbf{u} \cdot \mathbf{n}-\eta)+d^{I}\left(\frac{\partial \mathbf{u}}{\partial t} \cdot \mathbf{n}-\frac{\partial \eta}{\partial t}\right)\right. \\
& \left.\left.\quad+m^{I} \frac{\partial^{2} \mathbf{u}}{\partial t^{2}} \cdot \mathbf{n}-\frac{\partial^{2} \eta}{\partial t^{2}}\right)\right] \mathbf{n} \text { on } \Sigma .
\end{aligned}
$$

- Fluid

$$
\begin{aligned}
& \Delta p-\frac{1}{c^{2}} \frac{\partial^{2} p}{\partial t^{2}}=0 \text { in } \Omega_{\mathrm{F}}, \\
& \nabla p \cdot \mathbf{n}=-\rho_{\mathrm{F}} \frac{\partial^{2} \eta}{\partial t^{2}} \text { on } \Sigma .
\end{aligned}
$$

- Interface damping

$$
\begin{aligned}
-p= & k^{I}(\mathbf{u} \cdot \mathbf{n}-\eta)+d^{I}\left(\frac{\partial \mathbf{u}}{\partial t} \cdot \mathbf{n}-\frac{\partial \eta}{\partial t}\right) \\
& \left.+m^{I} \frac{\partial^{2} \mathbf{u}}{\partial t^{2}} \cdot \mathbf{n}-\frac{\partial^{2} \eta}{\partial t^{2}}\right) \text { on } \Sigma .
\end{aligned}
$$

\subsection{Variational formulation in $(\mathbf{u}, \eta, p)$}

In this subsection, the variational formulation of the problem is obtained using the test-function method. For this purpose, we introduce the spaces $C_{u}, C_{\eta}$ and $C_{p}$ of sufficiently smooth functions associated to the field variables $\mathbf{u}, \eta$ and $p$, respectively.

- Let $\delta \mathbf{u}$ be the time-independent test function, associated to $\mathbf{u}$, belonging to the admissible space $C_{u}^{*}=\left\{\delta \mathbf{u} \in C_{u} \mid \delta \mathbf{u}=\mathbf{0}\right.$ on $\left.\Gamma_{u}\right\}$. Multiplying Eq. (7a) by $\delta \mathbf{u} \in C_{u}^{*}$, applying a Green's formula, and taking Eqs. (7b) and (7d) into account, we have:

$$
\begin{gathered}
\int_{\Omega_{\mathrm{S}}} \operatorname{tr}[\boldsymbol{\sigma}(\mathbf{u}) \boldsymbol{\varepsilon}(\delta \mathbf{u})] \mathrm{d} x-k^{I} \int_{\Sigma} \eta \mathbf{n} \cdot \delta \mathbf{u} \mathrm{d} \sigma+k^{I} \int_{\Sigma}(\mathbf{u} \cdot \mathbf{n}) \mathbf{n} \cdot \delta \mathbf{u} \mathrm{d} \sigma \\
-d^{I} \int_{\Sigma} \frac{\partial \eta}{\partial t} \mathbf{n} \cdot \delta \mathbf{u} \mathrm{d} \sigma+d^{I} \int_{\Sigma}\left(\frac{\partial \mathbf{u}}{\partial t} \cdot \mathbf{n}\right) \mathbf{n} \cdot \delta \mathbf{u} \mathrm{d} \sigma \\
-m^{I} \int_{\Sigma} \frac{\partial^{2} \eta}{\partial t^{2}} \mathbf{n} \cdot \delta \mathbf{u} \mathrm{d} \sigma+m^{I} \int_{\Sigma}\left(\frac{\partial^{2} \mathbf{u}}{\partial t^{2}} \cdot \mathbf{n}\right) \mathbf{n} \cdot \delta \mathbf{u} \mathrm{d} \sigma \\
+\int_{\Omega_{\mathrm{S}}} \rho_{\mathrm{S}} \frac{\partial^{2} \mathbf{u}}{\partial t^{2}} \cdot \delta \mathbf{u} \mathrm{d} x=\int_{\Gamma_{\mathrm{t}}} \mathbf{F}^{\mathrm{d}} \cdot \delta \mathbf{u} \mathrm{d} \sigma .
\end{gathered}
$$

- Similarly, let $\delta p$ be the time-independent test function, associated to $p$, belonging to the admissible space $C_{p}$. Multiplying Eq. (8a) by $\delta p \in C_{p}$, applying a Green's formula, and taking Eq. (8b) into account, we obtain:

$$
\begin{aligned}
& \frac{1}{\rho_{\mathrm{F}}} \int_{\Omega_{\mathrm{F}}} \nabla p \cdot \nabla \delta p \mathrm{~d} x+\frac{1}{\rho_{\mathrm{F}} c^{2}} \int_{\Omega_{\mathrm{F}}} \frac{\partial^{2} p}{\partial t^{2}} \delta p \mathrm{~d} x \\
& \quad+\int_{\Sigma} \frac{\partial^{2} \eta}{\partial t^{2}} \delta p \mathrm{~d} \sigma=0 .
\end{aligned}
$$

- Finally, let $\delta \eta$ be the time-independent test function, associated to $\eta$, belonging to the admissible space $C_{\eta}$. Multiplying Eq. (9) by $\delta \eta \in C_{\eta}$, we have:

$$
\begin{gathered}
k^{I} \int_{\Sigma} \eta \delta \eta \mathrm{d} \sigma-k^{I} \int_{\Sigma} \mathbf{u} \cdot \mathbf{n} \delta \eta \mathrm{d} \sigma+d^{I} \int_{\Sigma} \frac{\partial \eta}{\partial t} \delta \eta \mathrm{d} \sigma-d^{I} \int_{\Sigma} \frac{\partial \mathbf{u}}{\partial t} \cdot \mathbf{n} \delta \eta \mathrm{d} \sigma \\
+m^{I} \int_{\Sigma} \frac{\partial^{2} \eta}{\partial t^{2}} \delta \eta \mathrm{d} \sigma-m^{I} \int_{\Sigma}\left(\frac{\partial^{2} \mathbf{u}}{\partial t^{2}} \cdot \mathbf{n}\right) \delta \eta \mathrm{d} \sigma-\int_{\Sigma} p \delta \eta \mathrm{d} \sigma=0
\end{gathered}
$$

Thus, the variational unsymmetric formulation of the elastoacoustic problem with interface damping consists, for given appropriate initial conditions, in finding $(\mathbf{u}, \eta, p) \in\left(C_{u}^{*}, C_{\eta}, C_{p}\right)$ such that, $\forall(\delta \mathbf{u}, \delta \eta, \delta p) \in\left(C_{u}^{*}, C_{\eta}, C_{p}\right)$, Eqs. (10)-(12) are satisfied.

\subsection{Finite element formulation}

After discretizing by the finite element method the bilinear forms in Eqs. (10)-(12), we obtain the following matrix equation of the coupled system:

$$
\begin{aligned}
& \left(\begin{array}{ccc}
\mathbf{M}_{u}+m^{I} \mathbf{D}_{u} & -m^{I} \mathbf{C}_{u \eta} & \mathbf{0} \\
-m^{I} \mathbf{C}_{u \eta}^{T} & m^{I} \mathbf{D}_{\eta} & \mathbf{0} \\
\mathbf{0} & \mathbf{C}_{\eta p}^{T} & \mathbf{M}_{p}
\end{array}\right)\left(\begin{array}{c}
\ddot{\mathbf{U}} \\
\ddot{\mathbf{H}} \\
\ddot{\mathbf{P}}
\end{array}\right) \\
& +\left(\begin{array}{ccc}
d^{I} \mathbf{D}_{u} & -d^{I} \mathbf{C}_{u \eta} & \mathbf{0} \\
-d^{I} \mathbf{C}_{u \eta}^{T} & d^{I} \mathbf{D}_{\eta} & \mathbf{0} \\
\mathbf{0} & \mathbf{0} & \mathbf{0}
\end{array}\right)\left(\begin{array}{c}
\dot{\mathbf{U}} \\
\dot{\mathbf{H}} \\
\dot{\mathbf{P}}
\end{array}\right) \\
& +\left(\begin{array}{ccc}
\mathbf{K}_{u}+k^{I} \mathbf{D}_{u} & -k^{I} \mathbf{C}_{u \eta} & \mathbf{0} \\
-k^{I} \mathbf{C}_{u \eta}^{T} & k^{I} \mathbf{D}_{\eta} & -\mathbf{C}_{\eta p} \\
\mathbf{0} & \mathbf{0} & \mathbf{K}_{p}
\end{array}\right)\left(\begin{array}{l}
\mathbf{U} \\
\mathbf{H} \\
\mathbf{P}
\end{array}\right)=\left(\begin{array}{l}
\mathbf{F} \\
\mathbf{0} \\
\mathbf{0}
\end{array}\right)
\end{aligned}
$$

where $\mathbf{U}, \mathbf{H}$ and $\mathbf{P}$ are the vectors of nodal values of $\mathbf{u}, \eta$ and $p$, respectively; $\mathbf{F}$ is the vector of external forces defined by $\int_{\Gamma_{\mathrm{t}}} \mathbf{F}^{\mathrm{d}} \cdot \delta \mathbf{u} \mathrm{d} \sigma \Rightarrow \delta \mathbf{U}^{\mathrm{T}} \mathbf{F}$; and the submatrices of Eq. (13) are given by the set of Eq. (14) defined as follows:

$$
\begin{aligned}
& \int_{\Omega_{\mathrm{S}}} \operatorname{tr}[\boldsymbol{\sigma}(\mathbf{u}) \boldsymbol{\varepsilon}(\delta \mathbf{u})] \mathrm{d} x \Rightarrow \delta \mathbf{U}^{\mathrm{T}} \mathbf{K}_{u} \mathbf{U}, \quad \int_{\Omega_{\mathrm{S}}} \rho_{\mathrm{S}} \frac{\partial^{2} \mathbf{u}}{\partial t^{2}} \cdot \delta \mathbf{u} \mathrm{d} x \Rightarrow \delta \mathbf{U}^{\mathrm{T}} \mathbf{M}_{u} \ddot{\mathbf{U}}, \\
& \frac{1}{\rho_{\mathrm{F}}} \int_{\Omega_{\mathrm{F}}} \nabla p \cdot \nabla \delta p \mathrm{~d} x \Rightarrow \delta \mathbf{P}^{\mathrm{T}} \mathbf{K}_{p} \mathbf{P}, \quad \frac{1}{\rho_{\mathrm{F}} c^{2}} \int_{\Omega_{\mathrm{F}}} \frac{\partial^{2} p}{\partial t^{2}} \delta p \mathrm{~d} x \Rightarrow \delta \mathbf{P}^{\mathrm{T}} \mathbf{M}_{p} \ddot{\mathbf{P}}, \\
& \int_{\Sigma} p \delta \eta \mathrm{d} \sigma \Rightarrow \delta \mathbf{H}^{\mathrm{T}} \mathbf{C}_{\eta p} \mathbf{P}, \quad \int_{\Sigma} \frac{\partial^{2} \eta}{\partial t^{2}} \delta p \mathrm{~d} \sigma \Rightarrow \delta \mathbf{P}^{\mathrm{T}} \mathbf{C}_{\eta p}^{\mathrm{T}} \ddot{\mathbf{H}}, \\
& \int_{\Sigma}(\mathbf{u} \cdot \mathbf{n}) \mathbf{n} \cdot \delta \mathbf{u} \mathrm{d} \sigma \Rightarrow \delta \mathbf{U}^{\mathrm{T}} \mathbf{D}_{u} \mathbf{U}, \quad \int_{\Sigma} \eta \delta \eta \mathrm{d} \sigma \Rightarrow \delta \mathbf{H}^{\mathrm{T}} \mathbf{D}_{\eta} \mathbf{H}, \\
& \int_{\Sigma} \eta \mathbf{n} \cdot \delta \mathbf{u} \mathrm{d} \sigma \Rightarrow \delta \mathbf{U}^{\mathrm{T}} \mathbf{C}_{u \eta} \mathbf{H}, \quad \int_{\Sigma} \mathbf{u} \cdot \mathbf{n} \delta \eta \mathrm{d} \sigma \Rightarrow \delta \mathbf{H}^{\mathrm{T}} \mathbf{C}_{u \eta}^{\mathrm{T}} \mathbf{U} .
\end{aligned}
$$




\subsection{Spectral fluid-structure problem with interface damping in $(\mathbf{u}, \eta, p)$}

In order to compute the natural vibration modes of the coupled system with interface damping, we consider harmonic solutions for the structure displacement $\mathbf{u}$, normal fluid displacement at the interface $\eta$, and fluid pressure $p$. In other terms, we assume the following form for $\mathbf{u}, \eta$ and $p$ :

$\mathbf{u}(x, t)=\mathbf{u}(x) \exp (-\mathrm{i} \omega t)$ for $x \in \Omega_{\mathrm{S}}, \quad t \geqslant 0$,

$\eta(x, t)=\eta(x) \exp (-\mathrm{i} \omega t)$ for $x \in \Sigma, \quad t \geqslant 0$,

$p(x, t)=p(x) \exp (-\mathrm{i} \omega t)$ for $x \in \Omega_{\mathrm{F}}, \quad t \geqslant 0$,

where $\omega$ is the angular frequency.

Substituting these expressions in Eqs. (10)-(12), the variational formulation of the elastoacoustic spectral problem with interface damping consists in finding $\omega \in \mathbb{C}$ and $(\mathbf{u}, \eta, p) \in\left(C_{u}^{*}, C_{\eta}, C_{p}\right)$, such that $\forall(\delta \mathbf{u}, \delta \eta, \delta p) \in\left(C_{u}^{*}, C_{\eta}, C_{p}\right)$ :

$$
\begin{aligned}
& \int_{\Omega_{\mathrm{S}}} \operatorname{tr}[\boldsymbol{\sigma}(\mathbf{u}) \boldsymbol{\varepsilon}(\delta \mathbf{u})] \mathrm{d} x+\left(k^{I}-i \omega d^{I}-\omega^{2} m^{I}\right) \\
& {\left[\int_{\Sigma}(\mathbf{u} \cdot \mathbf{n}) \mathbf{n} \cdot \delta \mathbf{u} \mathrm{d} \sigma-\int_{\Sigma} \eta \mathbf{n} \cdot \delta \mathbf{u} \mathrm{d} \sigma\right]-\omega^{2} \int_{\Omega_{\mathrm{S}}} \rho_{\mathrm{S}} \mathbf{u} \cdot \delta \mathbf{u} \mathrm{d} x=0,}
\end{aligned}
$$$$
\frac{1}{\rho_{\mathrm{F}}} \int_{\Omega_{\mathrm{F}}} \nabla p \cdot \nabla \delta p \mathrm{~d} x-\frac{\omega^{2}}{\rho_{\mathrm{F}} c^{2}} \int_{\Omega_{\mathrm{F}}} p \delta p \mathrm{~d} x-\omega^{2} \int_{\Sigma} \eta \delta p \mathrm{~d} \sigma=0
$$

$\left(k^{I}-i \omega d^{I}-\omega^{2} m^{I}\right)\left[\int_{\Sigma} \eta \delta \eta \mathrm{d} \sigma-\int_{\Sigma} \mathbf{u} \cdot \mathbf{n} \delta \eta \mathrm{d} \sigma\right]-\int_{\Sigma} p \delta \eta \mathrm{d} \sigma=0$.

In principle, one should use complex test-functions and sesquilinear products involving $(\overline{\delta \mathbf{u}}, \overline{\delta p}, \overline{\delta \eta})$ complex conjugate quantities of $(\delta \mathbf{u}, \delta p, \delta \eta)$. As we are concerned by finite element discretization, real test-functions have been introduced instead.

In discretized form, the previous variational formulation can be written as

$$
\begin{gathered}
{\left[\left(\begin{array}{ccc}
\mathbf{K}_{u}+k^{I} \mathbf{D}_{u} & -k^{I} \mathbf{C}_{u \eta} & \mathbf{0} \\
-k^{I} \mathbf{C}_{u \eta}^{\mathrm{T}} & k^{I} \mathbf{D}_{\eta} & -\mathbf{C}_{\eta p} \\
\mathbf{0} & \mathbf{0} & \mathbf{K}_{p}
\end{array}\right)-\mathrm{i} \omega\left(\begin{array}{ccc}
d^{I} \mathbf{D}_{u} & -d^{I} \mathbf{C}_{u \eta} & \mathbf{0} \\
-d^{I} \mathbf{C}_{u \eta}^{\mathrm{T}} & d^{I} \mathbf{D}_{\eta} & \mathbf{0} \\
\mathbf{0} & \mathbf{0} & \mathbf{0}
\end{array}\right)\right.} \\
\left.-\omega^{2}\left(\begin{array}{ccc}
\mathbf{M}_{u}+m^{I} \mathbf{D}_{u} & -m^{I} \mathbf{C}_{u \eta} & \mathbf{0} \\
-m^{I} \mathbf{C}_{u \eta}^{T} & m^{I} \mathbf{D}_{\eta} & \mathbf{0} \\
\mathbf{0} & \mathbf{C}_{\eta p}^{\mathrm{T}} & \mathbf{M}_{p}
\end{array}\right)\right]\left(\begin{array}{l}
\mathbf{U} \\
\mathbf{H} \\
\mathbf{P}
\end{array}\right)=\left(\begin{array}{l}
\mathbf{0} \\
\mathbf{0} \\
\mathbf{0}
\end{array}\right)
\end{gathered}
$$

or, in condensed form:

$\left(\mathscr{K}-\mathrm{i} \omega \mathscr{D}-\omega^{2} \mathscr{M}\right) \mathbf{X}=\mathbf{0}$.

It is important to note that this matrix system is not symmetric. The symmetrization, which will be detailed in Section 3, can be carried out by using an additional scalar variable to describe the fluid.

In order to solve the complex eigenvalue problem (19), let us transform it into a real problem of double size by complementing it with the equality $\lambda \mathscr{M} \mathbf{X}-\lambda \mathscr{M} \mathbf{X}=\mathbf{0}$ where $\lambda=-\mathrm{i} \omega$. The extended spectral equation takes the canonical form:

$(\mathbf{A}+\lambda \mathbf{B}) \mathbf{Y}=\mathbf{0}$

with the matrices:

$\mathbf{A}=\left(\begin{array}{cc}\mathscr{K} & \mathbf{0} \\ \mathbf{0} & -\mathscr{M}\end{array}\right) \quad \mathbf{B}=\left(\begin{array}{cc}\mathscr{D} & \mathscr{M} \\ \mathscr{M} & \mathbf{0}\end{array}\right)$

and the state vector:
$\mathbf{Y}=\left(\begin{array}{c}\mathbf{X} \\ \lambda \mathbf{X}\end{array}\right)$

\section{Remarks}

- Classical fluid-structure problem without damping

The natural vibration modes of the fluid-structure problem without interface damping is obtained by taking $m^{I}=d^{I}=0$ in Eq. (18). In this case, $\mathbf{H}$ can be expressed in terms of $\mathbf{U}$ and $\mathbf{P}$ from the second line of Eq. (18):

$\mathbf{H}=\mathbf{D}_{\eta}^{-1} \mathbf{C}_{u \eta}^{\mathrm{T}} \mathbf{U}+\frac{1}{k^{l}} \mathbf{D}_{\eta}^{-1} \mathbf{C}_{\eta p} \mathbf{P}$

Replacing this expression into the first and third lines of Eq. (18), we obtain the following matrix system in terms of $\mathbf{U}$ and $\mathbf{P}$ :

$$
\begin{aligned}
& {\left[\left(\begin{array}{c|c}
\mathbf{K}_{u}+k^{I}\left(\mathbf{D}_{u}-\mathbf{C}_{u \eta} \mathbf{D}_{\eta}^{-1} \mathbf{C}_{u \eta}^{T}\right) & -\mathbf{C}_{u p} \\
\hline \mathbf{0} & \mathbf{K}_{p}
\end{array}\right)\right.} \\
& \left.-\omega^{2}\left(\begin{array}{c|c}
\mathbf{M}_{u} & \mathbf{0} \\
\hline \mathbf{C}_{u p}^{T} & \frac{1}{k^{I}} \mathbf{C}_{\eta p}^{T} \mathbf{D}_{\eta}^{-1} \mathbf{C}_{\eta p}+\mathbf{M}_{p}
\end{array}\right)\right]\left(\begin{array}{l}
\mathbf{U} \\
\mathbf{P}
\end{array}\right)=\left(\begin{array}{l}
\mathbf{0} \\
\mathbf{0}
\end{array}\right),
\end{aligned}
$$

where $\mathbf{C}_{u p}=\mathbf{C}_{u \eta} \mathbf{D}_{\eta}^{-1} \mathbf{C}_{\eta p}$.

Eq. (24) represents the matrix equation of a fluid-structure problem with a spring at the interface. Moreover, it can be noted that $\mathbf{D}_{u}=\mathbf{C}_{u \eta} \mathbf{D}_{\eta}^{-1} \mathbf{C}_{u \eta}^{\mathrm{T}}$. Therefore, if $k^{I}$ tends to infinity, the matrix equation of the coupled system can be written in the following form:

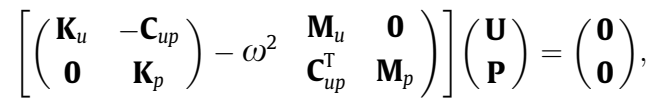

which corresponds to the standard unsymmetric system associated to the undamped structural-acoustic problem (see, e.g., [11]).

- Spectral fluid-structure problem with interface damping in $(\mathbf{u}, p)$ and $Z(\omega)$

Identifying the wall acoustic impedance $Z(\omega)=d^{I}-\mathrm{i}\left(\omega m^{I}-\right.$ $\left.k^{I} / \omega\right)$ in Eq. (18), leads to the following matrix equation:

$$
\begin{gathered}
{\left[\left(\begin{array}{ccc}
\mathbf{K}_{u} & \mathbf{0} & \mathbf{0} \\
\mathbf{0} & \mathbf{0} & -\mathbf{C}_{\eta p} \\
\mathbf{0} & \mathbf{0} & \mathbf{K}_{p}
\end{array}\right)-\mathrm{i} \omega Z(\omega)\left(\begin{array}{ccc}
\mathbf{D}_{u} & -\mathbf{C}_{u \eta} & \mathbf{0} \\
-\mathbf{C}_{u \eta}^{\mathrm{T}} & \mathbf{D}_{\eta} & \mathbf{0} \\
\mathbf{0} & \mathbf{0} & \mathbf{0}
\end{array}\right)\right.} \\
\left.-\omega^{2}\left(\begin{array}{ccc}
\mathbf{M}_{u} & \mathbf{0} & \mathbf{0} \\
\mathbf{0} & \mathbf{0} & \mathbf{0} \\
\mathbf{0} & \mathbf{C}_{\eta p}^{\mathrm{T}} & \mathbf{M}_{p}
\end{array}\right)\right]\left(\begin{array}{l}
\mathbf{U} \\
\mathbf{H} \\
\mathbf{P}
\end{array}\right)=\left(\begin{array}{l}
\mathbf{0} \\
\mathbf{0} \\
\mathbf{0}
\end{array}\right) .
\end{gathered}
$$

From the second line of Eq. (26), $\mathbf{H}$ can be expressed in terms of $\mathbf{U}$ and P:

$\mathbf{H}=\frac{-1}{\mathrm{i} \omega Z(\omega)} \mathbf{D}_{\eta}^{-1} \mathbf{C}_{\eta p} \mathbf{P}+\mathbf{D}_{\eta}^{-1} \mathbf{C}_{u \eta}^{\mathrm{T}} \mathbf{U}$

Replacing this expression into the first and third lines of Eq. (26) and taking into account the relation $\mathbf{D}_{u}=\mathbf{C}_{u \eta} \mathbf{D}_{\eta}^{-1} \mathbf{C}_{u \eta}^{\mathrm{T}}$, we obtain the following matrix system in terms of $\mathbf{U}$ and $\mathbf{P}$ :

$\left.\left[\left(\begin{array}{cc}\mathbf{K}_{u} & -\mathbf{C}_{u p} \\ \mathbf{0} & \mathbf{K}_{p}\end{array}\right)-\frac{\mathrm{i} \omega}{Z(\omega)}\left(\begin{array}{cc}\mathbf{0} & \mathbf{0} \\ \mathbf{0} & \mathbf{D}_{p}\end{array}\right)-\omega^{2} \quad \begin{array}{cc}\mathbf{M}_{u} & \mathbf{0} \\ \mathbf{C}_{u p}^{\mathrm{T}} & \mathbf{M}_{p}\end{array}\right)\right]\left(\begin{array}{l}\mathbf{U} \\ \mathbf{P}\end{array}\right)=\left(\begin{array}{l}\mathbf{0} \\ \mathbf{0}\end{array}\right)$,

where $\mathbf{D}_{p}=\mathbf{C}_{\eta p}^{\mathrm{T}} \mathbf{D}_{\eta}^{-1} \mathbf{C}_{\eta p}$.

Note that this eigenvalue problem is non-quadratic in terms of $\omega$ due to the rational fraction $\mathrm{i} \omega / Z(\omega)$. 


\section{Symmetric formulation for the spectral problem with interface damping}

In Section 2.7, an unsymmetric quadratic eigenvalue system has been obtained. The aim of this section is to establish a symmetric formulation of the structural-acoustic problem with interface damping in view of a direct treatment by finite elements.

Before describing the procedure, we will discuss the interest of introducing symmetric matrix system for this damped structural acoustic problem. As shown at the end of Section 4 (Eq. (46)), this coupled problem based on a scalar field description for the fluid, is of the type $\left(\mathscr{K}-\mathrm{i} \omega \mathscr{D}-\omega^{2} \mathscr{M}\right) \mathbf{X}=\mathbf{0}$ (or $=\mathbf{F}$ for prescribed forces) with real symmetric and frequency independent matrices where $\mathscr{K} \geqslant 0$ and $\mathscr{M}>0$. It should be noted that this problem corresponds to a classical structural dynamic problem in the time domain.

The aim is to build reduced order models using a basis projection on the associated conservative problem $\left(\mathscr{K}-\omega^{2} \mathscr{M}\right) \mathbf{X}=\mathbf{0}$, leading to a system of the type $\left(\mathscr{K}_{\text {red }}-\mathrm{i} \omega \mathscr{D}_{\text {red }}-\omega^{2} \mathscr{M}_{\text {red }}\right) \mathbf{X}_{\text {red }}=\mathbf{0}$ ( or $=\mathbf{F}_{\text {red }}$ for prescribed forces).

The eigenvalue problem $\left(\mathscr{K}-\omega^{2} \mathscr{M}\right) \mathbf{X}=\mathbf{0}$ can be dealt with classical eigenvalue solvers. It can be also solved, for a huge number of degrees-of-freedom, using a dynamic substructuring type method adapted to fluid-structure interaction problem as done in [11] (Chapter 9).

The reduced order system, because the number of degrees-offreedom is not anymore prohibitive, can be solved (i) either by inverting the matrix frequency by frequency for $\omega \in \mathbb{R}$ in the forced response case, (ii) or using a quadratic eigenvalue solver to obtain an appropriate basis constituted of complex eigenvectors (see e.g. $[14,15]$ ).

In the present paper, we have only checked the methodology on small sized systems using the Matlab quadratic eigensolver polyeig [15]. Of course, in practice, the interest of symmetrization lies in the construction of symmetric reduced order models, which is the subject of current investigations by the authors.

\subsection{Spectral boundary value problem in terms of $(\mathbf{u}, \eta, \varphi, p)$}

The symmetrization is obtained through the introduction of an intermediate unknown field [11], namely the fluid displacement potential field $\varphi$. Let us recall that this variable is defined up to an additive constant, such that $\mathbf{u}^{\mathrm{F}}=\nabla \varphi$.

Considering the redundant description of the fluid by using both pressure and displacement potential, the local equations of the spectral problem can be written in the form:

\section{- Structure}

$$
\begin{aligned}
& \operatorname{div} \boldsymbol{\sigma}(\mathbf{u})+\omega^{2} \rho_{\mathrm{S}} \mathbf{u}=\mathbf{0} \text { in } \Omega_{\mathrm{S}}, \\
& \boldsymbol{\sigma}(\mathbf{u}) \mathbf{n}^{\mathrm{S}}=\mathbf{0} \text { on } \Gamma_{t}, \\
& \mathbf{u}=\mathbf{0} \text { on } \Gamma_{u}, \\
& \boldsymbol{\sigma}(\mathbf{u}) \mathbf{n}^{\mathrm{S}}=-\left(k^{I}-\mathrm{i} \omega d^{I}-\omega^{2} m^{I}\right)(\mathbf{u} \cdot \mathbf{n}-\eta) \mathbf{n} \text { on } \Sigma .
\end{aligned}
$$

- Fluid

$$
\begin{aligned}
& \rho_{\mathrm{F}} \Delta \varphi+\frac{p}{c^{2}}=0 \text { in } \Omega_{\mathrm{F}}, \\
& \frac{\partial \varphi}{\partial n}=\eta \text { on } \Sigma, \\
& \frac{p}{\rho_{\mathrm{F}} c^{2}}=\frac{\omega^{2}}{c^{2}} \varphi \text { in } \Omega_{\mathrm{F}} .
\end{aligned}
$$

- Interface

$$
-\omega^{2} \rho_{\mathrm{F}} \varphi=\left(k^{I}-\mathrm{i} \omega d^{I}-\omega^{2} m^{I}\right)(\mathbf{u} \cdot \mathbf{n}-\eta) \text { on } \Sigma .
$$

\subsection{Variational formulation in $(\mathbf{u}, \eta, \varphi, p)$}

As in the unsymmetric case, we proceed in this subsection to the variational formulation of the problem by the test-function method:

- Multiplying Eq. (29a) by $\delta \mathbf{u} \in C_{u}^{*}$, integrating by parts (using Green's formula) and taking the boundary conditions (Eqs. (29b) and (29d)) into account, we have:

$$
\begin{aligned}
& \int_{\Omega_{\mathrm{S}}} \operatorname{tr}[\boldsymbol{\sigma}(\mathbf{u}) \boldsymbol{\varepsilon}(\delta \mathbf{u})] \mathrm{d} x+\left(k^{I}-\mathrm{i} \omega d^{I}-\omega^{2} m^{I}\right) \\
& {\left[\int_{\Sigma}(\mathbf{u} \cdot \mathbf{n}) \mathbf{n} \cdot \delta \mathbf{u} \mathrm{d} \sigma-\int_{\Sigma} \eta \mathbf{n} \cdot \delta \mathbf{u} \mathrm{d} \sigma\right]-\omega^{2} \int_{\Omega_{\mathrm{S}}} \rho_{\mathrm{S}} \mathbf{u} \cdot \delta \mathbf{u} \mathrm{d} x=0 .}
\end{aligned}
$$

- Similarly, we consider the space $C_{\varphi}$ of sufficiently regular function $\varphi$ defined on $\Omega_{\mathrm{F}}$. Multiplying Eq. (30a) by an arbitrary test function $\delta \varphi \in C_{\varphi}$ and taking Eq. (30b) into account, we obtain:

$$
-\rho_{\mathrm{F}} \int_{\Omega_{\mathrm{F}}} \nabla \varphi \cdot \nabla \delta \varphi \mathrm{d} x+\rho_{\mathrm{F}} \int_{\Sigma} \eta \delta \varphi \mathrm{d} \sigma+\frac{1}{c^{2}} \int_{\Omega_{\mathrm{F}}} p \delta \varphi \mathrm{d} x=0 .
$$

- Then, multiplying Eq. (30c) by a test function $\delta p \in C_{p}$, we have:

$$
\frac{1}{\rho_{\mathrm{F}} c^{2}} \int_{\Omega_{\mathrm{F}}} p \delta p \mathrm{~d} x-\frac{\omega^{2}}{c^{2}} \int_{\Omega_{\mathrm{F}}} \varphi \delta p \mathrm{~d} x=0
$$

- Finally, multiplying Eq. (31) by a test function $\delta \eta \in C_{\eta}$, we obtain:

$$
\begin{aligned}
& \left(k^{I}-\mathrm{i} \omega d^{I}-\omega^{2} m^{I}\right)\left[\int_{\Sigma} \eta \delta \eta \mathrm{d} \sigma-\int_{\Sigma} \mathbf{u} \cdot \mathbf{n} \delta \eta \mathrm{d} \sigma\right] \\
& -\omega^{2} \rho_{\mathrm{F}} \int_{\Sigma} \varphi \delta \eta \mathrm{d} \sigma=0
\end{aligned}
$$

Thus, the symmetric variational formulation of the elastoacoustic problem with interface damping consists in finding $\omega \in \mathbb{C}$ and $(\mathbf{u}, \varphi, \eta, p) \in\left(C_{u}^{*}, C_{\varphi}, C_{\eta}, C_{p}\right)$ such that $\forall(\delta \mathbf{u}, \delta \varphi, \delta \eta, \delta p) \in\left(C_{u}^{*}, C_{\varphi}\right.$, $\left.C_{\eta}, C_{p}\right)$, Eqs. (32)-(35) are satisfied.

\subsection{Finite element formulation}

In the following, the symmetric problem, defined by Eqs. (32)(35), is discretized in order to obtain the corresponding matrix equations. Let us consider meshes in $\Omega_{\mathrm{F}}$ and $\Omega_{\mathrm{S}}$ with coinciding nodes at the interface. By using a proper finite element approximation of the variables, we obtain:

$$
\begin{gathered}
{\left[\left(\begin{array}{cccc}
\mathbf{K}_{u}+k^{I} \mathbf{D}_{u} & -k^{I} \mathbf{C}_{u \eta} & \mathbf{0} & \mathbf{0} \\
-k^{I} \mathbf{C}_{u \eta}^{\mathrm{T}} & k^{I} \mathbf{D}_{\eta} & \mathbf{0} & \mathbf{0} \\
\mathbf{0} & \mathbf{0} & \mathbf{M}_{p} & \mathbf{0} \\
\mathbf{0} & \mathbf{0} & \mathbf{0} & \mathbf{0}
\end{array}\right)-\mathrm{i} \omega\left(\begin{array}{cccc}
d^{I} \mathbf{D}_{u} & -d^{I} \mathbf{C}_{u \eta} & \mathbf{0} & \mathbf{0} \\
-d^{I} \mathbf{C}_{u \eta}^{\mathrm{T}} & d^{I} \mathbf{D}_{\eta} & \mathbf{0} & \mathbf{0} \\
\mathbf{0} & \mathbf{0} & \mathbf{0} & \mathbf{0} \\
\mathbf{0} & \mathbf{0} & \mathbf{0} & \mathbf{0}
\end{array}\right)\right.} \\
\left.-\omega^{2}\left(\begin{array}{cccc}
\mathbf{M}_{u}+m^{I} \mathbf{D}_{u} & -m^{I} \mathbf{C}_{u \eta} & \mathbf{0} & \mathbf{0} \\
-m^{I} \mathbf{C}_{u \eta}^{\mathrm{T}} & m^{I} \mathbf{D}_{\eta} & \mathbf{0} & \mathbf{A} \\
\mathbf{0} & \mathbf{0} & \mathbf{0} & \mathbf{B} \\
\mathbf{0} & \mathbf{A}^{\mathrm{T}} & \mathbf{B}^{\mathrm{T}} & -\mathbf{K}_{\varphi}
\end{array}\right)\right]\left(\begin{array}{l}
\mathbf{U} \\
\mathbf{H} \\
\mathbf{P} \\
\mathbf{\Phi}
\end{array}\right)=\left(\begin{array}{l}
\mathbf{0} \\
\mathbf{0} \\
\mathbf{0} \\
\mathbf{0}
\end{array}\right),
\end{gathered}
$$

where $\mathbf{U}, \mathbf{H}, \mathbf{P}$ and $\boldsymbol{\Phi}$ are the vectors of nodal values of $\mathbf{u}, \eta, p$ and $\varphi$ respectively, and where the not yet defined submatrices of Eq. (36) are given by the set of Eq. (37) defined as follows: 
$\rho_{\mathrm{F}} \int_{\Omega_{\mathrm{F}}} \nabla \varphi \cdot \nabla \delta \varphi \mathrm{d} x \Rightarrow \delta \mathbf{\Phi}^{\mathrm{T}} \mathbf{K}_{\varphi} \mathbf{\Phi}$,

$\rho_{\mathrm{F}} \int_{\Sigma} \varphi \delta \eta \mathrm{d} \sigma \Rightarrow \delta \mathbf{H}^{\mathrm{T}} \mathbf{A} \boldsymbol{\Phi}$

$\frac{1}{c^{2}} \int_{\Sigma} \varphi \delta p \mathrm{~d} \sigma \Rightarrow \delta \mathbf{P}^{\mathrm{T}} \mathbf{B} \boldsymbol{\Phi}$.

It should be noted that we have used the matrix notation $\mathbf{A}$ and $\mathbf{B}$ instead of the more natural ones $\mathbf{C}_{\eta \varphi}$ and $\mathbf{C}_{p \varphi}$ into the previous equations for sake of compactness in the sequel of the paper.

The fourth equation of the matrix system (36) allows us to eliminate the degrees-of-freedom associated with $\varphi$ following the rigorous condensation procedure described in [11]. This procedure consists of an a posteriori elimination of $\varphi$ in order to obtain an eigenvalue problem only in terms of $(\mathbf{u}, \eta, p)$. This procedure will be detailed in the next section.

\section{Symmetric matrix system resulting from the elimination of fluid variable}

In this section, we propose to eliminate the fluid displacement potential in the symmetric formulation (Eq. (36)) using the procedure described by Morand and Ohayon for undamped elastoacoustic problems [11].

Note that a special procedure, which will be detailed below, must be carried out because in general case, the matrix $\mathbf{K}_{\varphi}$ is singular.

In a first step, let us define a partitioning of $\boldsymbol{\Phi}$ by letting $\boldsymbol{\Phi}^{\mathrm{T}}=\left[\begin{array}{ll}\Phi_{1} & \boldsymbol{\Phi}_{2}^{\mathrm{T}}\end{array}\right]$ where $\Phi_{1}$ denotes a particular component of $\boldsymbol{\Phi}$ (here, the first), which induces the following partitioning of the matrices A, B and $\mathbf{K}_{\varphi}$ in Eq. (36):

$$
\begin{aligned}
& \left(\begin{array}{ccccc}
\mathbf{K}_{u}+k^{I} \mathbf{D}_{u} & -k^{I} \mathbf{C}_{u \eta} & \mathbf{0} & \mathbf{0} & \mathbf{0} \\
-k^{I} \mathbf{C}_{u \eta} & k^{I} \mathbf{D}_{\eta} & \mathbf{0} & \mathbf{0} & \mathbf{0} \\
\mathbf{0} & \mathbf{0} & \mathbf{M}_{p} & \mathbf{0} & \mathbf{0} \\
\mathbf{0} & \mathbf{0} & \mathbf{0} & 0 & \mathbf{0} \\
\mathbf{0} & \mathbf{0} & \mathbf{0} & \mathbf{0} & \mathbf{0}
\end{array}\right)\left(\begin{array}{c}
\mathbf{U} \\
\mathbf{H} \\
\mathbf{P} \\
\Phi_{1} \\
\boldsymbol{\Phi}_{2}
\end{array}\right) \\
& -\mathrm{i} \omega\left(\begin{array}{ccccc}
d^{I} \mathbf{D}_{u} & -d^{I} \mathbf{C}_{u \eta} & \mathbf{0} & \mathbf{0} & \mathbf{0} \\
-d^{I} \mathbf{C}_{u \eta}^{\mathrm{T}} & d^{I} \mathbf{D}_{\eta} & \mathbf{0} & \mathbf{0} & \mathbf{0} \\
\mathbf{0} & \mathbf{0} & \mathbf{0} & \mathbf{0} & \mathbf{0} \\
\mathbf{0} & \mathbf{0} & \mathbf{0} & 0 & \mathbf{0} \\
\mathbf{0} & \mathbf{0} & \mathbf{0} & \mathbf{0} & \mathbf{0}
\end{array}\right)\left(\begin{array}{c}
\mathbf{U} \\
\mathbf{H} \\
\mathbf{P} \\
\Phi_{1} \\
\boldsymbol{\Phi}_{2}
\end{array}\right) \\
& -\omega^{2}\left(\begin{array}{ccccc}
\mathbf{M}_{u}+m^{I} \mathbf{D}_{u} & -m^{I} \mathbf{C}_{u \eta} & \mathbf{0} & \mathbf{0} & \mathbf{0} \\
-m^{I} \mathbf{C}_{u \eta} & m^{I} \mathbf{D}_{\eta} & \mathbf{0} & \mathbf{A}_{1} & \mathbf{A}_{2} \\
\mathbf{0} & \mathbf{0} & \mathbf{0} & \mathbf{B}_{1} & \mathbf{B}_{2} \\
\mathbf{0} & \mathbf{A}_{1}^{\mathrm{T}} & \mathbf{B}_{1}^{\mathrm{T}} & -K_{\varphi 11} & -\mathbf{K}_{\varphi 12} \\
\mathbf{0} & \mathbf{A}_{2}^{\mathrm{T}} & \mathbf{B}_{2}^{\mathrm{T}} & -\mathbf{K}_{\varphi 21} & -\mathbf{K}_{\varphi 22}
\end{array}\right)\left(\begin{array}{c}
\mathbf{U} \\
\mathbf{H} \\
\mathbf{P} \\
\Phi_{1} \\
\boldsymbol{\Phi}_{2}
\end{array}\right) \\
& =\left(\begin{array}{l}
\mathbf{0} \\
\mathbf{0} \\
\mathbf{0} \\
0 \\
\mathbf{0}
\end{array}\right)
\end{aligned}
$$

In a second step, as $\mathbf{K}_{\varphi 22}$ is nonsingular, $\boldsymbol{\Phi}_{2}$ can be expressed in terms of $\mathbf{P}, \mathbf{H}$ and $\Phi_{1}$ using the fifth line of Eq. (38):

$\boldsymbol{\Phi}_{2}=-\mathbf{K}_{\varphi 22}^{-1} \mathbf{K}_{\varphi 21} \Phi_{1}+\mathbf{K}_{\varphi 22}^{-1} \mathbf{A}_{2}^{\mathrm{T}} \mathbf{H}+\mathbf{K}_{\varphi 22}^{-1} \mathbf{B}_{2}^{\mathrm{T}} \mathbf{P}$

Substituting this relation for the remaining, the fourth line of Eq. (38) becomes:

$$
\begin{aligned}
& \left(\mathbf{A}_{1}^{\mathrm{T}}-\mathbf{K}_{\varphi 12} \mathbf{K}_{\varphi 22}^{-1} \mathbf{A}_{2}^{\mathrm{T}}\right) \mathbf{H}+\left(\mathbf{B}_{1}^{\mathrm{T}}-\mathbf{K}_{\varphi 12} \mathbf{K}_{\varphi 22}^{-1} \mathbf{B}_{2}^{\mathrm{T}}\right) \mathbf{P} \\
& =\left(K_{\varphi 11}-\mathbf{K}_{\varphi 12} \mathbf{K}_{\varphi 22}^{-1} \mathbf{K}_{\varphi 21}\right) \Phi_{1} .
\end{aligned}
$$

It can be shown that [11]:

$K_{\varphi 11}-\mathbf{K}_{\varphi 12} \mathbf{K}_{\varphi 22}^{-1} \mathbf{K}_{\varphi 12}^{\mathrm{T}}=0$.

Thus, Eq. (40) can be written as

$\mathbf{a}^{\mathrm{T}} \mathbf{H}+\mathbf{b}^{\mathrm{T}} \mathbf{P}=0$

where matrices $\mathbf{a}$ and $\mathbf{b}$ are defined by:

$\mathbf{a}^{\mathrm{T}}=\mathbf{A}_{1}^{\mathrm{T}}-\mathbf{K}_{\varphi 12} \mathbf{K}_{\varphi 22}^{-1} \mathbf{A}_{2}^{\mathrm{T}}$,

$\mathbf{b}^{\mathrm{T}}=\mathbf{B}_{1}^{\mathrm{T}}-\mathbf{K}_{\varphi 12} \mathbf{K}_{\varphi 22}^{-1} \mathbf{B}_{2}^{\mathrm{T}}$.

Finally, replacing $\boldsymbol{\Phi}_{2}$ by its expression (Eq. (39)) in the three first lines of Eq. (38) and using Eq. (42), the following matrix system is obtained:

$$
\begin{aligned}
& \left(\begin{array}{cccc}
\mathbf{K}_{u}+k^{I} \mathbf{D}_{u} & -k^{I} \mathbf{C}_{u \eta} & \mathbf{0} & \mathbf{0} \\
-k^{I} \mathbf{C}_{u \eta}^{\mathrm{T}} & k^{I} \mathbf{D}_{\eta} & \mathbf{0} & \mathbf{0} \\
\mathbf{0} & \mathbf{0} & \mathbf{M}_{p} & \mathbf{0} \\
\mathbf{0} & \mathbf{0} & \mathbf{0} & 0
\end{array}\right)\left(\begin{array}{c}
\mathbf{U} \\
\mathbf{H} \\
\mathbf{P} \\
\Phi_{1}
\end{array}\right) \\
& -\mathrm{i} \omega\left(\begin{array}{cccc}
d^{I} \mathbf{D}_{u} & -d^{I} \mathbf{C}_{u \eta} & \mathbf{0} & \mathbf{0} \\
-d^{I} \mathbf{C}_{u \eta}^{\mathrm{T}} & d^{I} \mathbf{D}_{\eta} & \mathbf{0} & \mathbf{0} \\
\mathbf{0} & \mathbf{0} & \mathbf{0} & \mathbf{0} \\
\mathbf{0} & \mathbf{0} & \mathbf{0} & 0
\end{array}\right)\left(\begin{array}{c}
\mathbf{U} \\
\mathbf{H} \\
\mathbf{P} \\
\Phi_{1}
\end{array}\right) \\
& -\omega^{2}\left(\begin{array}{ccccc}
\mathbf{M}_{u}+m^{I} \mathbf{D}_{u} & -m^{I} \mathbf{C}_{u \eta} & \mathbf{0}^{2} & \mathbf{0} \\
-m^{I} \mathbf{C}_{u \eta}^{\mathrm{T}} & m^{I} \mathbf{D}_{\eta}+\mathbf{A}_{2} \mathbf{K}_{\varphi 22}^{-1} \mathbf{A}_{2}^{\mathrm{T}} & \mathbf{A}_{2} \mathbf{K}_{\varphi 22}^{-1} \mathbf{B}_{2}^{\mathrm{T}} & \mathbf{a} \\
\mathbf{0} & \mathbf{B}_{2} \mathbf{K}_{\varphi 22}^{-1} \mathbf{A}_{2}^{\mathrm{T}} & \mathbf{B}_{2} \mathbf{K}_{\varphi 22}^{-1} \mathbf{B}_{2}^{\mathrm{T}} & \mathbf{b} \\
\mathbf{0} & \mathbf{a}^{\mathrm{T}} & 0
\end{array}\right)\left(\begin{array}{c}
\mathbf{U} \\
\mathbf{H} \\
\mathbf{P} \\
\Phi_{1}
\end{array}\right) \\
& =\left(\begin{array}{l}
\mathbf{0} \\
\mathbf{0} \\
\mathbf{0} \\
0
\end{array}\right) .
\end{aligned}
$$

The last equation of (44) can be written as

$-\omega^{2}\left(\mathbf{a}^{\mathrm{T}} \mathbf{H}+\mathbf{b}^{\mathrm{T}} \mathbf{P}\right)=0$,

which leads, for $\omega \neq 0$, to: $\mathbf{a}^{\mathrm{T}} \mathbf{H}+\mathbf{b}^{\mathrm{T}} \mathbf{P}=0$. Therefore, Eq. (44) can be viewed as the following matrix system, valid for $\omega=0$ :

$$
\begin{aligned}
& \left(\begin{array}{ccc}
\mathbf{K}_{u}+k^{I} \mathbf{D}_{u} & -k^{I} \mathbf{C}_{u \eta} & \mathbf{0} \\
-k^{I} \mathbf{C}_{u \eta}^{T} & k^{I} \mathbf{D}_{\eta} & \mathbf{0} \\
\mathbf{0} & \mathbf{0} & \mathbf{M}_{p}
\end{array}\right)\left(\begin{array}{l}
\mathbf{U} \\
\mathbf{H} \\
\mathbf{P}
\end{array}\right) \\
& -\mathrm{i} \omega\left(\begin{array}{ccc}
d^{I} \mathbf{D}_{u} & -d^{I} \mathbf{C}_{u \eta} & \mathbf{0} \\
-d^{I} \mathbf{C}_{u \eta}^{\mathrm{T}} & d^{I} \mathbf{D}_{\eta} & \mathbf{0} \\
\mathbf{0} & \mathbf{0} & \mathbf{0}
\end{array}\right)\left(\begin{array}{l}
\mathbf{U} \\
\mathbf{H} \\
\mathbf{P}
\end{array}\right) \\
& -\omega^{2}\left(\begin{array}{ccc}
\mathbf{M}_{u}+m^{I} \mathbf{D}_{u} & -m^{I} \mathbf{C}_{u \eta} & \mathbf{0} \\
-m^{I} \mathbf{C}_{u \eta}^{\mathrm{T}} & m^{I} \mathbf{D}_{\eta}+\mathbf{A}_{2} \mathbf{K}_{\varphi 22}^{-1} \mathbf{A}_{2}^{\mathrm{T}} & \mathbf{A}_{2} \mathbf{K}_{\varphi 22}^{-1} \mathbf{B}_{2}^{\mathrm{T}} \\
\mathbf{0} & \mathbf{B}_{2} \mathbf{K}_{\varphi 22}^{-1} \mathbf{A}_{2}^{\mathrm{T}} & \mathbf{B}_{2} \mathbf{K}_{\varphi 22}^{-1} \mathbf{B}_{2}^{\mathrm{T}}
\end{array}\right)\left(\begin{array}{l}
\mathbf{U} \\
\mathbf{H} \\
\mathbf{P}
\end{array}\right) \\
& =\left(\begin{array}{l}
\mathbf{0} \\
\mathbf{0} \\
\mathbf{0}
\end{array}\right)
\end{aligned}
$$

in which $\mathbf{H}$ and $\mathbf{P}$ must satisfy the constraint $\mathbf{a}^{\mathrm{T}} \mathbf{H}+\mathbf{b}^{\mathrm{T}} \mathbf{P}=0$. 


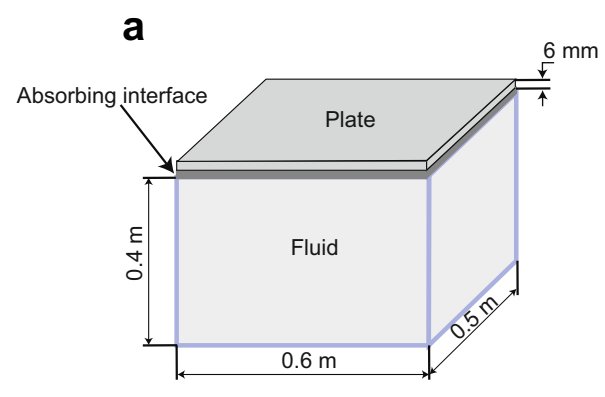

b

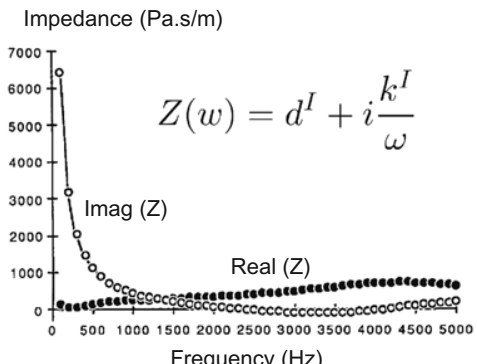

Fig. 3. Plate/acoustic cavity system: geometrical data and acoustic impedance.

\section{Remarks}

- In the last matrix system, of the form $\left(\mathscr{K}-\mathrm{i} \omega \mathscr{D}-\omega^{2} \mathscr{M}\right) \mathbf{X}=\mathbf{0}$, it can be shown that the matrices are real symmetric with $\mathscr{K} \geqslant 0$ and $\mathscr{M}>0$ (see Ref. [11]).

- The previous reduction is not an approximation but a rigorous condensation procedure.

- The constraint $\mathbf{a}^{\mathrm{T}} \mathbf{H}+\mathbf{b}^{\mathrm{T}} \mathbf{P}=0$ corresponds to the discretization of the variational property (33), for $\delta \varphi$ constant $(\delta \varphi=1)$. It results from the conservation of the total mass of fluid contained on $\Omega_{\mathrm{F}}$. It should also be noted that, in Eq. (44), $\Phi_{1}$ plays the role of a Lagrange multiplier.

\section{Numerical examples}

We present in this section some finite element results, obtained with the previous formulations, for the analysis of interior damped structural-acoustic systems. Firstly, a 3D free vibration elastoacoustic problem with damping interface is analyzed. Then, a comparison between the proposed approach and a full 3D modelling of the dissipative layer using Biot-Allard theory is presented. These two first examples are computed using the unsymmetric formulation proposed in Eq. (18). The resulting eigenvalue problem is solved using Matlab's polyeig function which is based on the algorithm described on page 267 of [15]. Finally, the third example concerns the vibration and transient analysis of a 2D rectangular tank completely filled with air. In this last case, the symmetric formulation (44) in the frequency domain and the unsymmetric formulation (13) in the time domain are employed. Note that even if it is symmetric, the quadratic eigenvalue problem (44) has been solved using the same algorithm as in the previous examples.

\subsection{Free vibration analysis of a $3 D$ plate/acoustic cavity system with} damping interface

We consider in this first example the spectral problem of a 3D rectangular acoustic cavity of size $A=0.6 \mathrm{~m}, B=0.5 \mathrm{~m}$ and $C=$ $0.4 \mathrm{~m}$ (see Fig. 3a) completely filled with air $\left(\rho_{\mathrm{F}}=1 \mathrm{~kg} / \mathrm{m}^{3}, c=\right.$ $340 \mathrm{~m} / \mathrm{s}$ ). One wall of the cavity is a flexible plate of thickness $6 \mathrm{~mm}$ clamped by its whole boundary and covered with a thin
Table 1

Frequencies $(\mathrm{Hz})$ of a 3D rigid acoustic cavity with an absorbing wall

\begin{tabular}{llllll}
\hline Undamped & & & Damped & & \\
\cline { 1 - 2 } \cline { 5 - 6 } 4096 d.o.f. & Exact & & 1452 d.o.f. & 4352 d.o.f. & Exact [13] \\
\hline 283.85 & $f_{100}=283.33$ & & $275.98-0.15 \mathrm{i}$ & $275.35-0.15 \mathrm{i}$ & $274.85-0.15 \mathrm{i}$ \\
340.62 & $f_{010}=340.00$ & & $330.81-0.23 \mathrm{i}$ & $330.06-0.23 \mathrm{i}$ & $329.46-0.23 \mathrm{i}$ \\
425.78 & $f_{001}=425.00$ & & $403.32-0.55 \mathrm{i}$ & $402.59-0.54 \mathrm{i}$ & $402.00-0.54 \mathrm{i}$ \\
443.39 & $f_{110}=442.58$ & & $429.45-0.46 \mathrm{i}$ & $428.48-0.46 \mathrm{i}$ & $427.71-0.46 \mathrm{i}$ \\
\hline
\end{tabular}

layer of absorbing material. The other walls are considered perfectly rigid. The mechanical parameters of the plate are: density $\rho_{\mathrm{S}}=7700 \mathrm{~kg} / \mathrm{m}^{3}$, Young's modulus $E=1.44 \times 10^{11} \mathrm{~Pa}$ and Poisson ratio $v=0.35$. The absorbing material, which is considered massless in this example, has two parameters: $k^{I}=5 \times 10^{6} \mathrm{~Pa} / \mathrm{m}$ and $\mathrm{d}^{I}=50 \mathrm{~Pa} . \mathrm{s} / \mathrm{m}$. These parameters are average impedance coefficients corresponding to a typical acoustic insulating fabric (a Johns Manville glass wool of thickness 1 inch) in the frequency range (50-500 Hz) (see Fig. 3b).

Firstly, we present the results obtained for the 3D acoustic cavity with and without damping interface. Table 1 gives the first four eigenfrequencies (in $\mathrm{Hz}$ ) with uniform meshes (hexagonal element) and with increasing number of degrees of freedom. The first and second columns present, respectively the frequencies of the rigid cavity computed from a pressure formulation and those obtained with the following exact solution:

$f_{m n k}=\frac{c}{2} \sqrt{\frac{m^{2}}{A^{2}}+\frac{n^{2}}{B^{2}}+\frac{k^{2}}{C^{2}}}$

The three other columns correspond to the complex frequencies of the damping cavity computed from the proposed formulation and compared to exact solution (last column) given in [13]. A good agreement between exact and computed values can be observed even for the coarse mesh. In this example, the imaginary part of the frequencies comes from dashpot dissipation. Moreover, the difference between the real part (damped case) and the real value (undamped case) of the frequencies is due to the spring effect. Figs. 4 and 5 show the pressure field in the acoustic cavity for the damped and undamped cases.
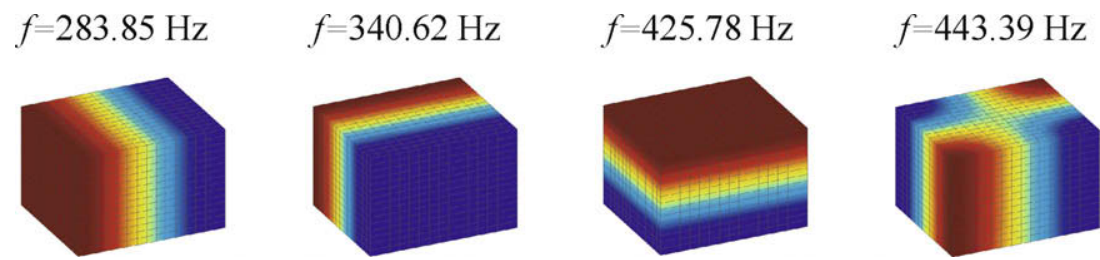

Fig. 4. First four acoustic modes for the 3D acoustic rigid cavity. 


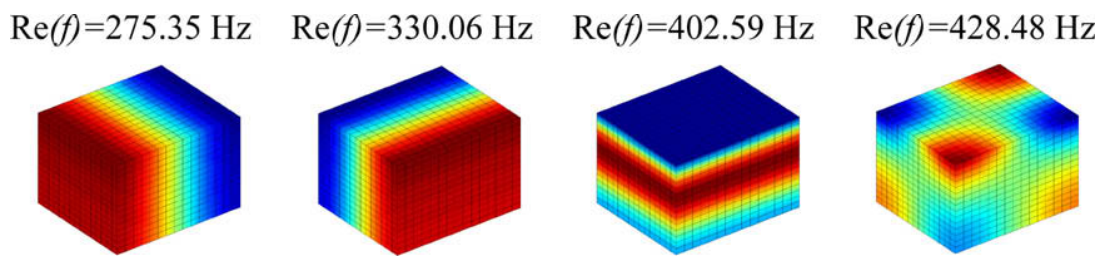

Fig. 5. First four acoustic modes for the 3D acoustic cavity with an absorbing wall.

Table 2

Computed frequencies $(\mathrm{Hz})$ of the structural-acoustic coupled system

\begin{tabular}{|c|c|c|c|c|c|c|}
\hline \multirow[t]{3}{*}{ Mode } & \multicolumn{4}{|l|}{ Undamped } & \multicolumn{2}{|l|}{ Damped } \\
\hline & F (i) & S (ii) & FSI (iii) & FSI [13] & FSI (iv) & FSI [13] \\
\hline & 4096 d.o.f. & 980 d.o.f. & 5076 d.o.f. & & 5332 d.o.f. & \\
\hline A & - & 158.13 & 158.18 & 156.61 & $158.18-0.00 \mathrm{i}$ & $156.91-0.00 \mathrm{i}$ \\
\hline B & 283.85 & - & 281.91 & 280.90 & $275.30-0.18 \mathrm{i}$ & $273.43-0.13 \mathrm{i}$ \\
\hline $\mathrm{C}$ & - & 290.24 & 291.95 & 294.37 & $291.75-0.00 \mathrm{i}$ & $294.07-0.01 \mathrm{i}$ \\
\hline $\mathrm{D}$ & 340.62 & - & 339.93 & 338.01 & $330.43-0.22 \mathrm{i}$ & $326.64-0.31 \mathrm{i}$ \\
\hline $\mathrm{E}$ & - & 362.83 & 363.19 & 375.80 & $375.80-0.01 \mathrm{i}$ & $375.97-0.01 \mathrm{i}$ \\
\hline $\mathrm{F}$ & 425.78 & - & 425.89 & 422.97 & $403.49-0.55 i$ & $394.04-1.30 \mathrm{i}$ \\
\hline G & 443.39 & - & 443.07 & 441.91 & $429.21-0.46 \mathrm{i}$ & $417.79-1.72 \mathrm{i}$ \\
\hline
\end{tabular}

Secondly, we consider the plate/acoustic-cavity system. Table 2 gives the eigenfrequencies in four cases: (i) 3D rigid acoustic cavity; (ii) clamped plate; (iii) plate/acoustic cavity coupled system without damping interface; and (iv) plate/acoustic cavity coupled system with damping interface. In the third and forth cases, our results are compared to those given in [13]. It should be noted that we have used, for the structural part, a four-node membraneshear-bending plate element (based on the first-order shear deformation theory) with five degrees-of-freedom per node and a selective reduced integration on the transverse shear.

The present results are in good agreement with those obtained in [13] with a displacement formulation for both domains (fluid and structure). As shown in this table, modes A, C and E correspond to the first three vibration modes of the structure (lower than $400 \mathrm{~Hz}$ ) and the four others (B, D, F and G) are the first four acoustic modes. In the damped case, notice that although the real parts of the frequencies corresponding to the structure modes remain practically unchanged, those associated with the fluid modes decrease between $2 \%$ and $10 \%$. The imaginary parts of the frequencies are almost zero for the structure modes, which means that they are only very slightly damped. As expected, the imaginary parts of the fluid modes are higher. Thus, the damping is stronger for the acoustic modes. For illustration purposes, Fig. 6 shows the deformed plate and the pressure field for the first four vibration modes in the coupled case.

5.2. Acoustic cavity with poroelastic treatment: comparison between the proposed approach and a full 3D modelling of the dissipative layer using Biot-Allard theory

In this example, we propose to compare the Kelvin-Voigt impedance approach with a full 3D modelling of the dissipative

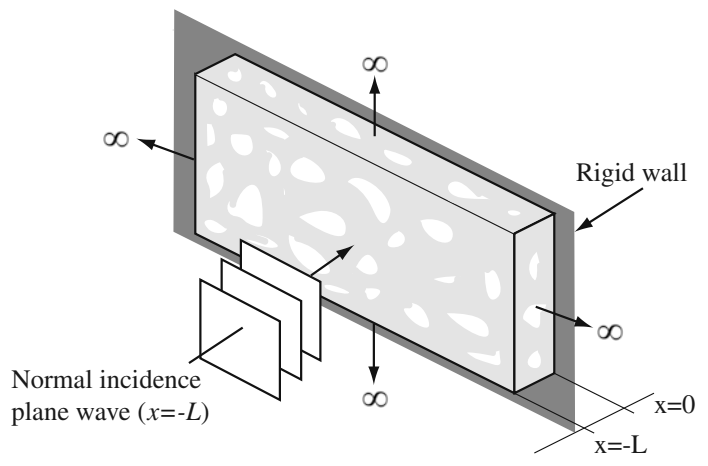

Fig. 7. Geometry of the single poroelastic layer surface impedance problem.

layer using Biot-Allard theory. Firstly, the Kelvin-Voigt impedance parameters $\left(k^{I}\right.$ and $\left.d^{I}\right)$ are determined from the surface impedance of laterally infinite poroelastic material computed using Biot-Allard theory. The approach developed in this work is then employed to compute the response of an acoustic cavity damped by a partial poroelastic treatment. In this case, comparisons with Biot-Allard 3D finite element calculations are given.

\subsubsection{Surface impedance for a single poroelastic layer}

The surface impedance of laterally infinite poroelastic material is investigated in this section. The configuration under study is depicted in Fig. 7. The porous layer, of thickness $L=7.62 \mathrm{~cm}$ and whose mechanical properties are given in [16], is bonded onto a rigid wall at $x=0$ and excited by a normal incidence plane wave of unit amplitude at $x=-L$. The poroelastic material used in this example has the properties of a typical foam.
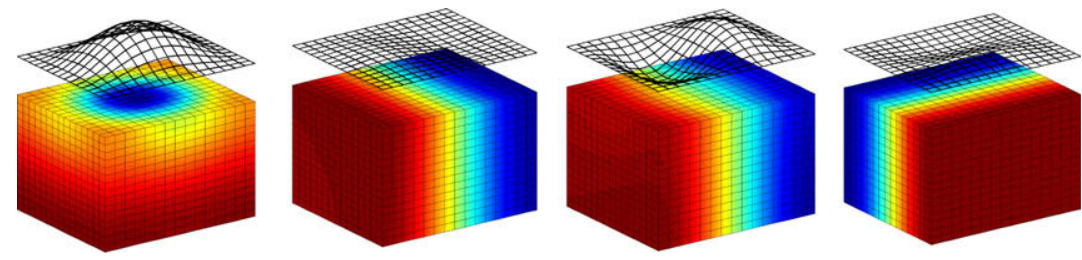

Fig. 6. First four modes without damping interface: fluid pressure level and plate total displacement. 

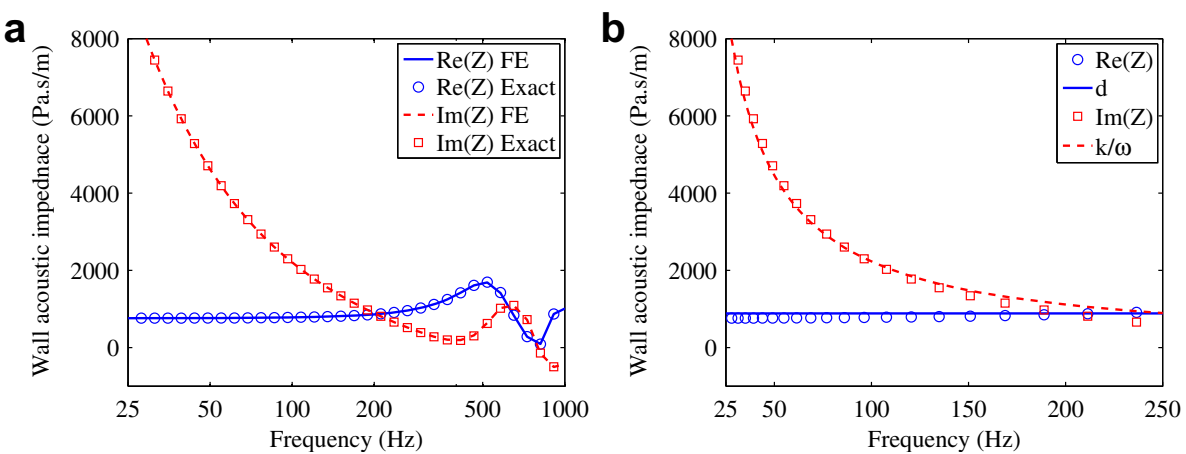

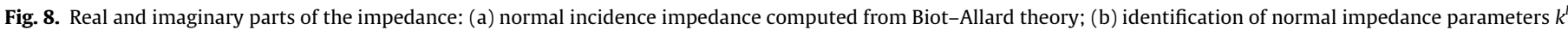
and $d^{l}$ in frequency range [25-250 Hz].

Note that the Biot-Allard model, whose detailed description can be found for example in [16], is defined by (i) five geometrical parameters (the porosity, the flow resistivity, the tortuosity, the viscous characteristic length, and the thermal characteristic length), (ii) the mechanical properties of the skeleton; and (iii) the saturating fluid properties.

The normal incidence impedance (Fig. 8) is calculated using the displacement of fluid $\left(\mathbf{u}^{\mathrm{f}}\right)$ and solid $\left(\mathbf{u}^{\mathrm{s}}\right)$ phases in the porous medium at the input surface $(x=-L)$ for the unit acoustic pressure excitation by the following equation:

$Z(\omega)=\frac{1}{\mathrm{i} \omega\left[\phi \mathbf{u}^{f}(-L)+(1-\phi) \mathbf{u}^{s}(-L)\right]}$,

where $\phi$ denotes the porosity of the material. In this equation, the displacement of fluid and solid phases are calculated from Biot-Allard theory using either analytical or finite element solutions (see, e.g., [3]).

In frequency range $[25-250 \mathrm{~Hz}]$, the normal incidence impedance can be approached by a Kelvin-Voigt model, i.e. sum of a constant real part and an imaginary part inversely proportional to the frequency:

$Z(\omega)=d^{I}+\mathrm{i} k^{I} / \omega$.

The parameters $k^{I}$ and $d^{I}\left(k^{I} \approx 1.4 \times 10^{6} \mathrm{~Pa} / \mathrm{m}\right.$ and $\left.d^{I} \approx 885 \mathrm{~Pa} \mathrm{~s} / \mathrm{m}\right)$, characterizing, respectively the elastic and the viscous aspect of the absorbing layer, are deduced from the acoustic surface impedance by a least squares method (Fig. 8 b).

\subsubsection{D enclosed cavity with porous absorbing material}

We consider now a 3D cubic enclosed cavity of $1 \mathrm{~m}^{3}$ filled with air (density $\rho_{0}=1 \mathrm{~kg} \mathrm{~m}^{-3}$, speed of sound $c_{0}=340 \mathrm{~m} \mathrm{~s}^{-1}$ ). The previously defined poroelastic material (see paragraph (5.2.1)) of size $0.6 \times 0.6 \times 0.0762 \mathrm{~m}^{3}$ is embedded to one wall of the cavity.

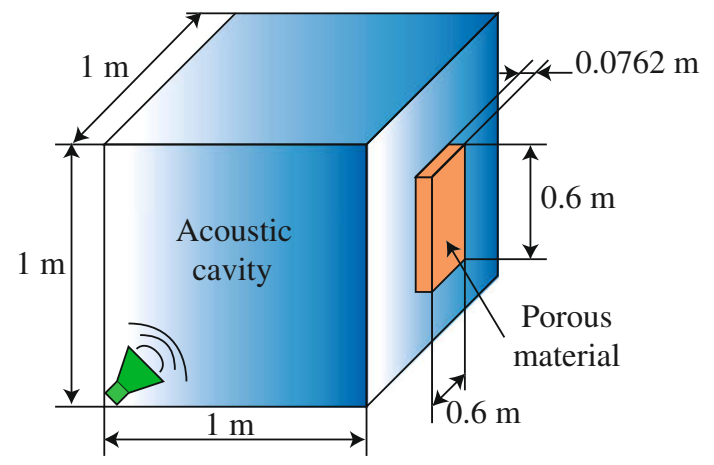

Fig. 9. Geometry of the 3D rigid cavity problem with porous absorbing material.
The cavity is excited by a volume velocity source placed in one corner $(x, y, z)=(0,0,0)$ (see Fig. 9). This example has been originally proposed by Panneton and Atalla [16].

The cavity is discretized using $10 \times 10 \times 10$ hexahedric elements with one degree-of-freedom per node corresponding to the acoustic pressure. In absence of the foam, this mesh is found to be fine enough for the frequency range of interest (see the comparison between numerically computed and exact eigenfrequencies given in Table 3). Moreover, using a 3D finite element Biot-Allard approach for the poroelastic domain, a $6 \times 6 \times 6$ mesh is employed.

The mean quadratic pressure in the closure is used as the indicator for this example:

$\left\langle p^{2}\right\rangle=\frac{1}{2 N_{a}} \sum_{i=1}^{N_{a}} p_{i}^{2}$

where $N_{a}$ is the number of nodes in the discretized acoustic domain and $p_{i}$ is the pressure in node $i$. In decibel $(\mathrm{dB})$, the associated pressure level is defined by

$L_{p}=10 \log \left(\frac{\left\langle p^{2}\right\rangle}{p_{0}^{2}}\right)$,

where $p_{0}=20 \mu \mathrm{Pa}$ is the reference sound pressure.

The results obtained with: (i) Biot-Allard finite element formulation, and (ii) the proposed Kelvin-Voigt localized impedance are analyzed and compared.

Fig. 10a presents the pressure level in the acoustic cavity with and without poroelastic treatment. The peaks observed correspond to the frequencies of the rigid acoustic cavity (see Table 3 ). This figure clearly shows that there is a substantial reduction in pressure level due to the addition of the poroelastic material.

The results obtained with the Biot-Allard 3D finite element formulation for the poroelastic treatment are compared to those obtained from the simplified Kelvin-Voigt impedance in Fig. 10b. An excellent agreement between the two methods is observed. This test validates the proposed approximations of the poroelastic medium by Kelvin-Voigt localized normal incidence impedance in low frequency range. It is worth noting that, in comparison with the Biot-Allard finite element formulation, the acoustic impedance approach is more efficient in terms of computational time and complexity. Indeed, with the Kelvin-Voigt approach, the porous

Table 3

Comparison between analytical and finite element frequencies of the acoustic rigid cavity

\begin{tabular}{llllllll}
\hline Exact $(\mathrm{Hz})$ & 170.07 & 240.52 & 294.58 & 340.15 & 380.30 & 416.60 & 481.05 \\
F.E. $(\mathrm{Hz})$ & 170.78 & 241.51 & 295.79 & 345.77 & 385.64 & 421.76 & 488.99 \\
\hline
\end{tabular}



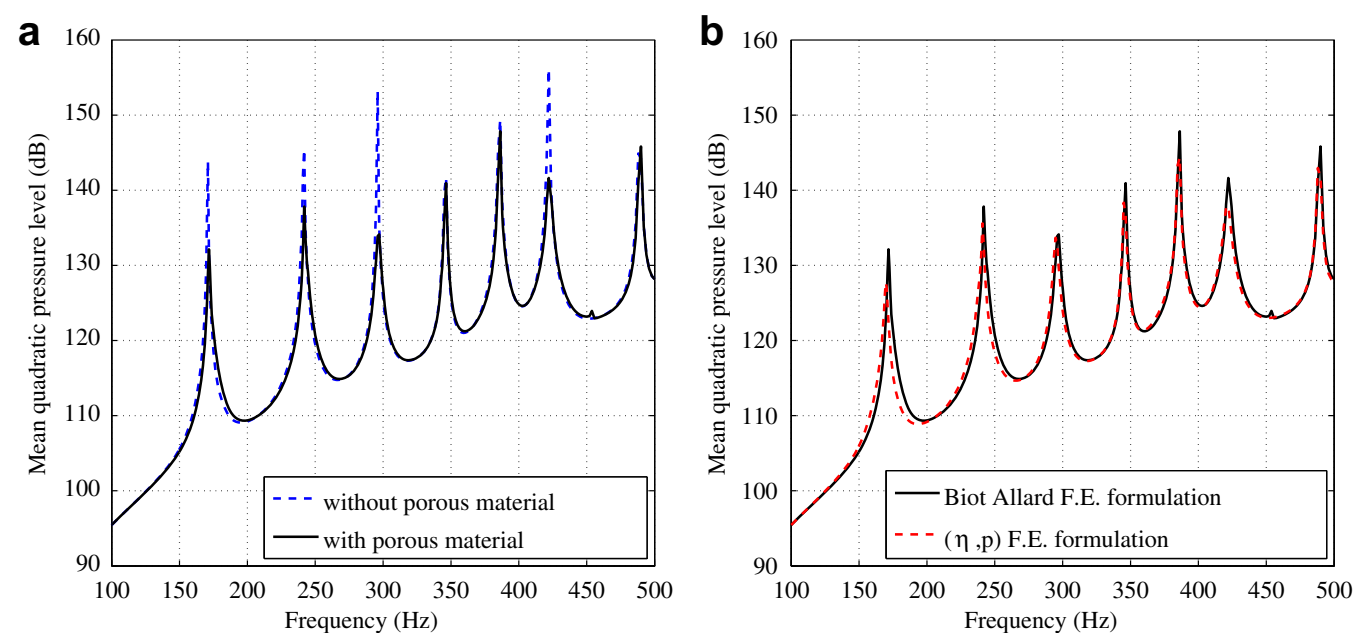

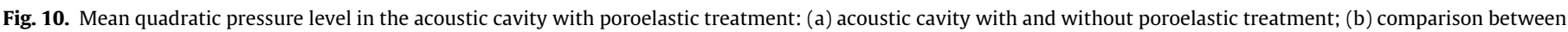
Biot-Allard and Kelvin-Voigt localized impedance finite element formulations.

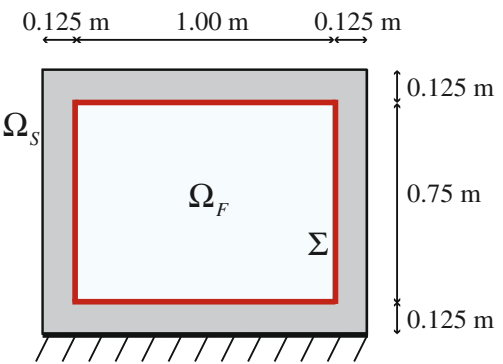

Fig. 11. Two-dimensional fluid-filled rectangular tank.

Table 4

Material parameters for the structure and the fluid

\begin{tabular}{lll}
\hline & Parameter & Value \\
\hline Structure & Density $\rho_{\mathrm{S}}\left(\mathrm{kg} / \mathrm{m}^{3}\right)$ & 7700 \\
& Young modulus $E(\mathrm{GPa})$ & 144 \\
\multirow{3}{*}{ Fluid } & Poisson ratio $v$ & 0.35 \\
& Density $\rho_{\mathrm{F}}\left(\mathrm{kg} / \mathrm{m}^{3}\right)$ & 1 \\
& Speed of sound $c(\mathrm{~m} / \mathrm{s})$ & 340 \\
\hline
\end{tabular}

treatment is modeled by interface elements and the problem can be solved directly in the time domain. However, if the poroelastic material is linked to a flexible wall, the performance of our simplified approach has to be confirmed. This point will be the subject of further investigations.

\subsection{Modal and transient analysis of a two-dimensional fluid-filled rectangular tank}

This third example concerns modal and transient analysis of a closed two-dimensional rectangular tank completely filled with air and covered by a thin layer of damping material whose parameters are the same as in the first example (Section 5.1). This example was initially proposed in [6].

The geometrical and physical data for the structure and the fluid are given in Fig. 11 and Table 4, respectively. Concerning the finite element discretization, linear quadrangular elements are used for the fluid and the structure with a compatible mesh at the fluidstructure interface.

Table 5 presents the eigenfrequencies in four cases: (i) rigid acoustic cavity; (ii) structure in vacuo; (iii) fluid-structure coupled system without damping interface; and (iv) fluid-structure cou-

Table 5

Computed frequencies $(\mathrm{Hz})$ of the structural-acoustic coupled system

\begin{tabular}{|c|c|c|c|c|c|c|}
\hline \multirow[t]{2}{*}{ Mode } & \multicolumn{4}{|c|}{ Undamped } & \multicolumn{2}{|l|}{ Damped } \\
\hline & $\mathrm{F}(\mathrm{i})$ & S (ii) & FSI (iii) & FSI [6] & FSI (iv) & FSI [6] \\
\hline A & - & 105.049 & 105.040 & - & $105.042-0.000 \mathrm{i}$ & - \\
\hline B & 170.132 & - & 170.132 & 169.930 & $158.128-0.109 \mathrm{i}$ & $158.431-0.060 \mathrm{i}$ \\
\hline C & 226.989 & - & 226.940 & 226.445 & $209.278-0.212 \mathrm{i}$ & $210.637-0.137 i$ \\
\hline $\mathrm{D}$ & 283.671 & - & 283.637 & 283.662 & $256.195-0.394 \mathrm{i}$ & $258.301-0.337 \mathrm{i}$ \\
\hline $\mathrm{E}$ & - & 305.690 & 305.665 & - & $305.605-0.003 \mathrm{i}$ & - \\
\hline $\mathrm{F}$ & 341.058 & - & 340.991 & 339.252 & $316.346-0.454 \mathrm{i}$ & $314.189-0.561 \mathrm{i}$ \\
\hline G & 409.689 & - & 409.600 & 408.990 & $369.690-0.829 \mathrm{i}$ & $362.014-1.594 \mathrm{i}$ \\
\hline
\end{tabular}

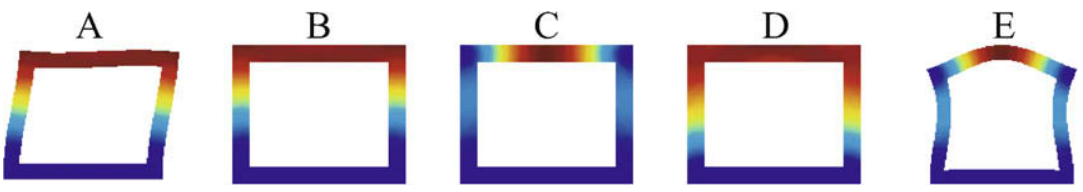

Fig. 12. Structure displacement for the first five eigenmodes of the coupled system without damping. 
A

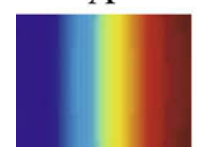

$\mathrm{B}$

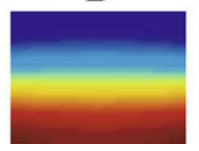

$\mathrm{C}$

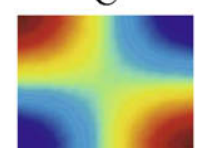

$\mathrm{D}$

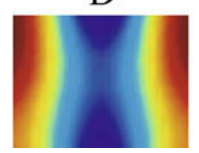

$\mathrm{E}$

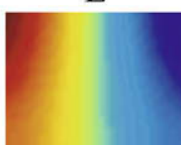

Fig. 13. Pressure level for the first five eigenmodes of the coupled system without damping.

Table 6

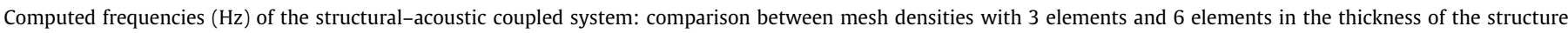

\begin{tabular}{|c|c|c|c|c|c|c|}
\hline \multirow[t]{2}{*}{ Mode } & \multicolumn{4}{|c|}{ Undamped } & \multicolumn{2}{|l|}{ Damped } \\
\hline & S (3 elt) & S (6 elt) & FSI (3 elt) & FSI (6 elt) & FSI (3 elt) & FSI (6 elt) \\
\hline A & 105.049 & 101.172 & 105.040 & 101.162 & $105.042-0.000 \mathrm{i}$ & $101.164-0.000 \mathrm{i}$ \\
\hline B & - & - & 170.132 & 170.043 & $158.128-0.109 \mathrm{i}$ & $158.049-0.109 \mathrm{i}$ \\
\hline $\mathrm{C}$ & - & - & 226.940 & 226.731 & $209.278-0.212 \mathrm{i}$ & $209.108-0.211 \mathrm{i}$ \\
\hline $\mathrm{D}$ & - & - & 283.637 & 283.418 & $256.195-0.394 \mathrm{i}$ & $255.994-0.393 \mathrm{i}$ \\
\hline $\mathrm{E}$ & 305.690 & 295.863 & 305.665 & 295.846 & $305.605-0.003 \mathrm{i}$ & $295.815-0.001 \mathrm{i}$ \\
\hline $\mathrm{F}$ & - & - & 340.991 & 340.279 & $316.346-0.454 \mathrm{i}$ & $315.675-0.454 \mathrm{i}$ \\
\hline G & - & - & 409.600 & 408.896 & $369.690-0.829 \mathrm{i}$ & $368.997-0.828 \mathrm{i}$ \\
\hline
\end{tabular}

pled system with damping interface. Note that in the third and forth cases, our results are compared to those given in [6].

The same kind of comments as those of the first example can be made here (see Section 5.1). For illustration purpose, Figs. 12 and 13 present the structure and fluid mode shapes in the undamped case.

Table 6 presents a comparison of our results for two mesh densities in the thickness of the structure: 3 and 6 finite elements. The frequencies for the structure in vacuo are compared with those given by the finite elements code Nastran using 20 Quad4 elements in the thickness. The results obtained with Nastran are $99.18 \mathrm{~Hz}$ for mode A and $291.47 \mathrm{~Hz}$ for mode E. Thus, the error between our results with a fine mesh ( 6 elements) and those given by Nastran is lower than $2 \%$. With only three elements, our results are not en-

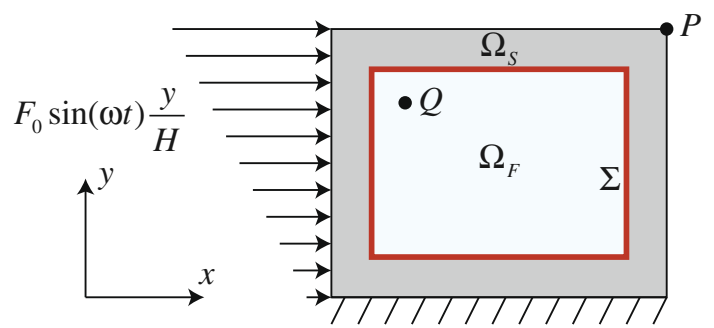

Fig. 14. Structural-acoustic system submitted to an harmonic excitation.

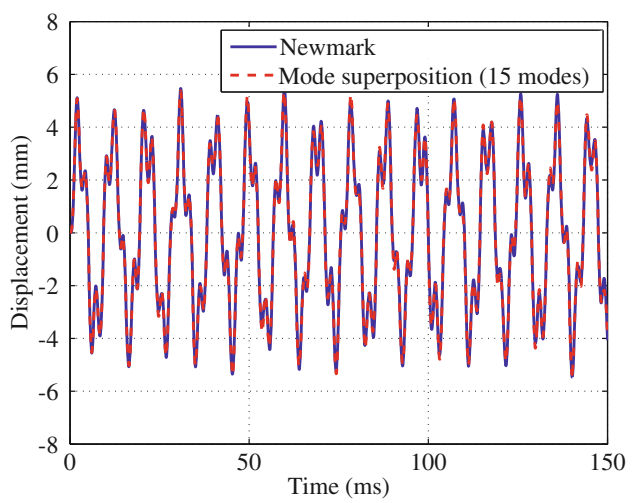

Fig. 15. Time-displacement response at point $P$ in the structure: comparison between direct time integration and mode superposition. ough accurate due to the fact that the structural bending is not properly computed. Thus, it is necessary to use a sufficiently fine mesh in the thickness of the structure. However, the acoustic modes of the coupled problem (modes B, C, D, F and G) remain practically unchanged because these first modes are less dependent on mesh refinement.

The structure is now excited at its left edge, of height $\mathrm{H}$, by a sinusoidal force which depends on the $y$-coordinate $F^{\mathrm{d}}=$ $F_{0} \sin (\omega t) y / H$ with $F_{0}=1000 \mathrm{~N}$ and $\omega=2 \pi \times 380 \mathrm{rad} / \mathrm{s}$ (see Fig. 14).

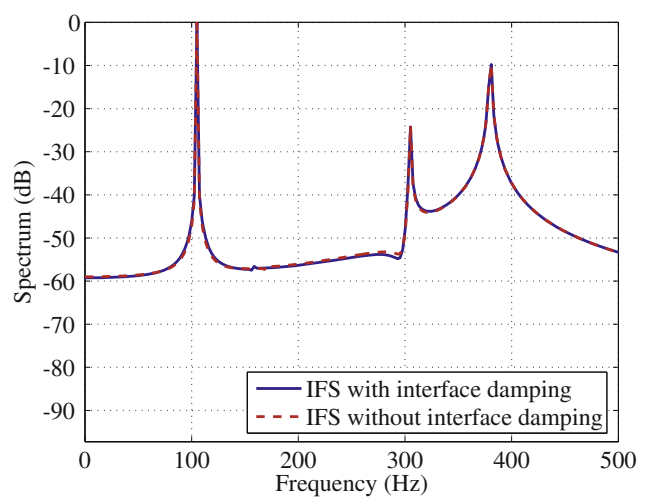

Fig. 16. Frequency response at point $P$ in the structure: comparison between responses of the coupled systems with and without damping interface.

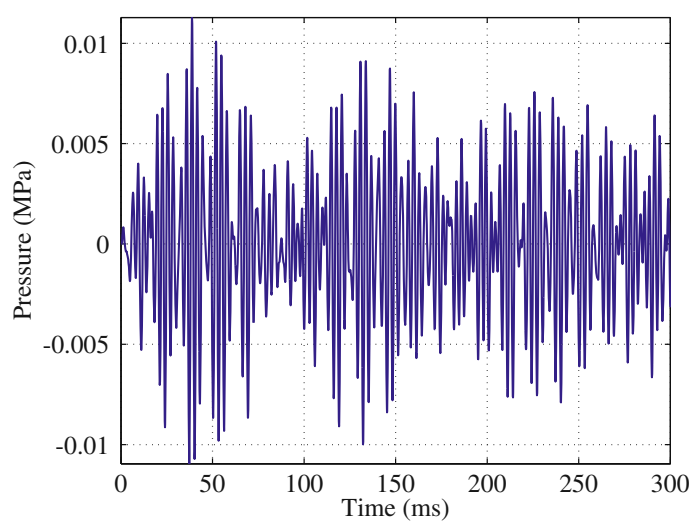

Fig. 17. Pressure versus time at point $Q$ in the fluid. 


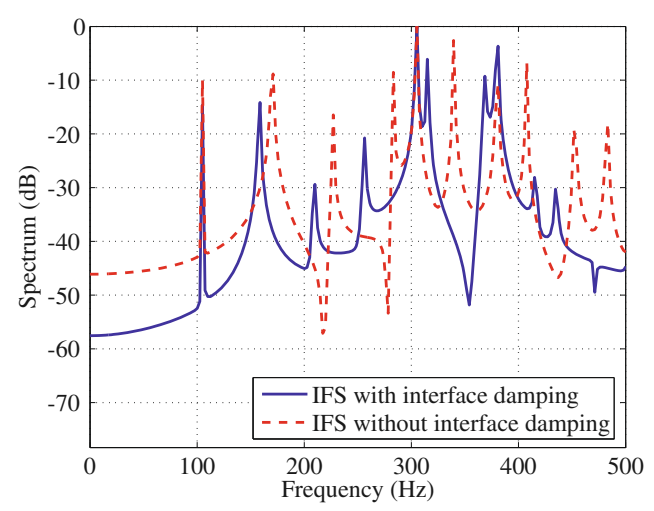

Fig. 18. Frequency response at point $Q$ in the fluid: comparison between responses of the coupled systems with and without damping interface.

For the resolution, we use a direct time integration method and a modal superposition technique. In the first case, the Newmark scheme is used for the time integration of the global unsymmetric system obtained via the $(\mathbf{u}, \eta, p)$ formulation. In such a case, the resolution becomes very expensive when the study time increases. In order to reduce the size of the problem, a modal superposition technique is employed. In this case, the symmetric formulation described in Section 3 is used in order to avoid a specific unsymmetric eigenvalue solver and the calculation of the left and right eigenvectors. This approach consists of projecting the system on a truncated basis using only some of the first modes. The reduced system can then be integrated in time in order to compute the transient response of the coupled fluid-structure system.

Fig. 15 presents the response of the coupled system with damping interface at point $P(1.25 \mathrm{~m}, 1.00 \mathrm{~m})$ of the structure. As can be seen, the response given by a modal superposition technique using only the first fifteen modes is similar to that one given by the integration of the global system using the Newmark scheme. Let us recall that the unsymmetric formulation in $(\mathbf{u}, \eta, p)$ is used for direct time integration method, and the symmetric formulation (after elimination of $\phi$ ) is used for the modal reduction.

Fig. 16 shows the frequency response of the coupled system at point $P$, with and without damping interface. As can be seen, the two curves are superposed in a frequency range from 0 to $500 \mathrm{~Hz}$. This means that the absorbing material do not affect the structural behavior of the vibroacoustic system. Moreover, three peaks can be observed in this graph. The first two peaks correspond to the first two vibration modes of the structure, and the third one corresponds to the excitation frequency.

Fig. 17 shows the time response of the coupled system with damping interface at point $Q(0.25 \mathrm{~m}, 0.75 \mathrm{~m})$ in the fluid. Here, we can observe the attenuation of the acoustic vibration amplitudes due to the damping effect.

Fig. 18 gives the frequency response of the coupled system at point $Q$ in the fluid, with and without damping interface. As can be seen, the frequencies of the acoustic modes are affected by the absorbing interface. It can be noted that all peaks correspond to the real part of the computed frequencies, except that one at $380 \mathrm{~Hz}$, which corresponds to the excitation frequency.

\section{Conclusion}

In this paper, a new finite element formulation for structuralacoustic systems with interface damping has been proposed. The coupled system consists of an elastic structure (described by its displacement field) containing an acoustic fluid (described by its pressure field). To take into account the effect of adding a thin layer of damping material at the fluid-structure, an additional unknown variable has been introduced, namely the normal fluid displacement field at the interface. With this new scalar unknown, various interface damping models can be introduced in the variational formulation and the associated finite element matrix system can be solved in frequency and time domains. In this paper, a simple Kelvin-Voigt model has been used to represent the interface damping. Then, the formulation has been written in a symmetric form through the introduction of an intermediate unknown field, namely the displacement potential of the fluid. Finally, numerical examples have been presented validating the new formulation and showing the influence of the damping model on the transient dynamic responses of structural-acoustic systems. Further analysis concerning hybrid passive/active treatments, which will coupled piezoelectric modeling $[17,18]$ and the dissipative modeling presented in this paper, are the subject of current investigations.

\section{Acknowledgement}

This research is partially supported by the European Community through the Marie Curie Research Training Network "A Computer Aided Engineering Approach to Smart Structures Design" under Contract No. MRTN-CT-2006-035559.

\section{References}

[1] J.-F. Allard, Propagation of Sound in Porous Media: Modelling Sound Absorbing Materials, Elsevier Applied Science, London, 1993.

[2] P. Göransson, A 3-D, symmetric, finite element formulation of the Biot equations with application to acoustic wave propagation through an elastic porous medium, Int. J. Numer. Methods Engrg. 41 (1) (1998) 167-192.

[3] P. Davidsson, Structure-acoustic analysis; Finite element modelling and reduction methods, Ph.D Thesis, Lund University, 2004.

[4] P. Davidsson, G. Sandberg, A reduction method for structure-acoustic and poroelastic-acoustic problems using interface-dependent Lanczos vectors, Comput. Methods Appl. Mech. Engrg. 195 (17-18) (2006) 1933-1945.

[5] V. Kehr-Candille, R. Ohayon, Elastoacoustic damped vibrations - finite element and modal reduction methods, in: O.C. Zienkiewicz, P. Ladevèze (Eds.), New Advances in Computational Structural Mechanics, Elsevier, Amsterdam, Netherlands, 1992, pp. 321-334.

[6] A. Bermúdez, R. Rodríguez, Modelling and numerical solution of elastoacoustic vibrations with interface damping, Int. J. Numer. Methods Engrg. 46 (10) (1999) 1763-1779.

[7] W. Larbi, J.-F. Deü, R. Ohayon, A new finite element formulation for internal acoustic problems with dissipative walls, Int. J. Numer. Methods Engrg. 68 (3) (2006) 381-399.

[8] J.-F. Deü, W. Larbi, R. Ohayon, Dissipative interface modeling for vibroacoustic problems - A new symmetric formulation, in: C.A. Mota Soares, J.A. Martins, H.C. Rodrigues, J.A.C. Ambrósio (Eds.), Computational Mechanics - Solids, Structures and Coupled Problems, Springer, Dordrecht, Netherlands, 2006, pp. 413-428.

[9] A.D. Pierce, Acoustics: An Introduction to Its Physical Principles and Applications, Acoustical Society of America, New York, 1989.

[10] R. Ohayon, Transient and modal analysis of bounded medium fluid-structure problems, in: R.W. Lewis, E. Hinton, P. Bettess, B.A. Schrefler (Eds.), Proceedings of the International Conference on Numerical Methods for Transient and Coupled Problems, Pineridge Press, 1984, pp. 139-149.

[11] H.J.-P. Morand, R. Ohayon, Fluid-Structure Interaction, Wiley, New York, 1995

[12] R. Ohayon, C. Soize, Structural Acoustics and Vibration, Academic Press, London, 1998.

[13] A. Bermúdez, L. Hervella-Nieto, R. Rodríguez, Finite element computation of the vibrations of a plate-fluid system with interface damping, Comput. Methods Appl. Mech. Engrg. 190 (24-25) (2001) 3021-3038.

[14] C. Conca, M. Duran, J. Planchard, A quadratic eigenvalue problem involving Stokes equations, Comput. Methods Appl. Mech. Engrg. 100 (3) (1992) 295313.

[15] F. Tisseur, K. Meerbergen, The quadratic eigenvalue problem, SIAM Rev. 41 (2) (2001) 235-286.

[16] R. Panneton, N. Atalla, An efficient finite element scheme for solving the threedimensional poroelasticity problem in acoustics, J. Acoust. Soc. Am. 101 (6) (1997) 3287-3298.

[17] J.-F. Deü, W. Larbi, R. Ohayon, Piezoelectric structural acoustic problems: symmetric variational formulations and finite element results, Comput. Methods Appl. Mech. Engrg. 197 (19-20) (2008) 1715-1724.

[18] W. Larbi, J.-F. Deü, R. Ohayon, Vibration of axisymmetric composite piezoelectric shells coupled with internal fluid, Int. J. Numer. Methods Engrg. 71 (12) (2007) 1412-1435. 